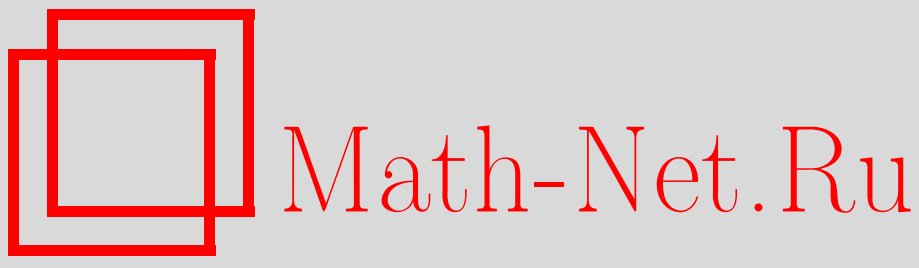

А. И. Булгаков, Л. И. Ткач, Возмущение выпуклозначного оператора многозначным отображением типа Гаммерштейна с невыпуклыми образами и краевые задачи для функционально-дифференциальных включений, Матем. сб., 1998, том 189, номер 6, 3-32

DOI: https://doi.org/10.4213/sm320

Использование Общероссийского математического портала Math-Net.Ru подразумевает, что вы прочитали и согласны с пользовательским соглашением

http://www . mathnet.ru/rus/agreement

Параметры загрузки:

IP: 18.209 .158 .208

26 апреля 2023 г., 12:22:24 
УДК 517.9

\author{
А.И. Булгаков, Л.И. Ткач
}

\title{
Возмущение выпуклозначного оператора многозначным отображением типа Гаммерштейна с невыпуклыми образами и краевые задачи для функционально-дифференциальных включений
}

В пространстве непрерьвных на отрезке $[a, b]$ вектор-фуннкций рассматривается функциональное включение, правая часть которого состоит из суммы вьпуклозначного многозначного отображения и произведения линейного интегрального оператора и многозначного отображения с вьпукльпи по переключению образами. Для данного включения получены оценки близости решения включения к наперед заданной непрерьвной вектор-функции. На основе этих оценок изучается структура множества решений включения. Доказана теоремы о плотности множества решений данного включения в множестве решений "овыпукленного" включения, а также для этого включения доказан "бэнг-бэнг" принцип. Затем эта теория применяется для исследования множеств решений краевых задач функционально-дифференциальных вк лючений с невыпуклой правой частью.

Библиография: 37 названий.

В работе рассматривается включение, правая часть которого представляет собой сумму выпуклозначного отображения и многозначного оператора типа Гаммерштейна, не обладаюшего свойством вьпуклости значений. Поэтому (см. [1]) оператор, порожденный правой частью такого включения, не является замкнутым, выпуклым оператором. В связи с этим традиционные методы исследований (теорема Какутани [2, гл. XVI, §5], принцип сжимающих отображений [3, §0.2]) здесь неприменимы. Изучение такого включения в работе проводится на основе теории непрерывных ветвей многозначных отображений с привлечением теоремы Майкла [4] и результата работы [5]. Для этого включения доказываются теоремы сушествования решений, а также изучается структура множества решений. Теория такого включения применяется затем для изучения краевых задач дифференциальных включений с многозначным вектор-функционалом.

Пусть $Y$ - банахово пространство с нормой $\|\cdot\| ; U \subset Y, \bar{U}$ - замыкание множества $U$; со $U$ - выпуклая оболочка множества $U$; $\overline{\operatorname{co}} U=\overline{\operatorname{co~} U}$; $\overline{\operatorname{ext}} U$ - замыкание множества крайних точек множества $U ;\|U\|_{Y} \equiv \sup \{\|x\|: x \in U\} ; 2^{Y}$ - множество всех непустых, ограниченных подмножеств пространства $Y ; \Omega(Y)$ - множество всех непустых, ограниченных, замкнутых, выпуклых подмножеств пространства $Y ; B_{Y}[u ; \delta]$ - открытьй шар пространства $Y$ с центром в точке $u \in Y$ и радиусом $\delta$. Пусть $\Phi_{1}, \Phi_{2} \subset Y$. Тогда

$$
h_{Y}^{+}\left[\Phi_{1} ; \Phi_{2}\right] \equiv \sup \left\{\rho_{Y}\left[y ; \Phi_{2}\right]: y \in \Phi_{1}\right\},
$$


где $\rho_{Y}[\cdot ; \cdot]-$ расстояние между точкой и множеством;

$$
h_{Y}\left[\Phi_{1} ; \Phi_{2}\right] \equiv \max \left\{h_{Y}^{+}\left[\Phi_{1} ; \Phi_{2}\right] ; h_{Y}^{+}\left[\Phi_{2} ; \Phi_{1}\right]\right\}
$$

- хаусдорфово расстояние между множествами $\Phi_{1}$ и $\Phi_{2} ; \Phi_{1}+\Phi_{2}=\{x+y$ : $\left.x \in \Phi_{1}, y \in \Phi_{2}\right\}$ - сумма множеств $\Phi_{1}$ и $\Phi_{2}$.

Пусть $X$ - банахово пространство, $U \subset X$. Рассмотрим многозначное отображение $\Phi: U \rightarrow 2^{Y}$. Будем говорить, что $\Phi$ полунепрерьвно сверху (снизу) в точке $x \in U$, если для любой последовательности $x_{i} \in U, i=1,2, \ldots$, сходящейся к $x$ в $X$, выполняется равенство

$$
\lim _{i \rightarrow \infty} h_{Y}^{+}\left[\Phi\left(x_{i}\right) ; \Phi(x)\right]=0 \quad\left(\lim _{i \rightarrow \infty} h_{Y}^{+}\left[\Phi(x) ; \Phi\left(x_{i}\right)\right]=0\right) .
$$

Если отображение $\Phi$ полунепрерывно сверху (снизу) в каждой точке множества $U$, то будем говорить, что $\Phi$ полунепрерывно сверху (снизу) на $U$.

Пусть $\mathbb{R}^{n}$ - пространство $n$-мерных вектор-столбцов с нормой $|\cdot| ; \operatorname{comp}\left[\mathbb{R}^{n}\right]-$ множество всех непустых компактов пространства $\mathbb{R}^{n}$. Пусть $\mathscr{U} \subset[a, b]$ - измеримое по Лебегу множество $(\mu(\mathscr{U})>0, \mu(\cdot)$ - мера Лебега). Введем следующие обозначения:

$L^{n}(\mathscr{U})$ - пространство функций $x: \mathscr{U} \rightarrow \mathbb{R}^{n}$ с суммируемыми по Лебегу компонентами и нормой

$$
\|x\|_{L^{n}(\mathscr{U})}=\int_{\mathscr{U}}|x(s)| d s ;
$$

$D^{n}[a, b]-$ пространство абсолютно непрерывных функций $x:[a, b] \rightarrow \mathbb{R}^{n}$ с нормой

$$
\|x\|_{D^{n}}=|x(a)|+\|\dot{x}\|_{L^{n}[a, b]} ;
$$

$C^{n}[a, b]-$ пространство непрерывных функций $x:[a, b] \rightarrow \mathbb{R}^{n}$ с нормой

$$
\|x\|_{C^{n}[a, b]}=\max \{|x(t)|: t \in[a, b]\}
$$

$C_{+}^{1}[a, b]-$ конус неотрицательных функций пространства $C^{1}[a, b]$.

Будем говорить, что множество $\Phi \subset L^{n}[a, b]$ выпукло по переключению, если для любых измеримых по Лебегу множеств $\mathscr{U}_{1}, \mathscr{U}_{2} \subset[a, b]$ таких, что $\mathscr{U}_{1} \cap \mathscr{U}_{2}=\varnothing$, $\mathscr{U}_{1} \cup \mathscr{U}_{2}=[a, b]$, и любых $x, y \in \Phi$ справедливо включение $\chi\left(\mathscr{U}_{1}\right) x+\chi\left(\mathscr{U}_{2}\right) y \in \Phi$, где $\chi(\cdot)$ - характеристическая функция соответствующих множеств. Обозначим через $\Pi\left[L^{n}[a, b]\right]\left(\Omega\left(\Pi\left[L^{n}[a, b]\right]\right)\right)$ множество всех непустых, замкнутых, ограниченных и выпуклых по переключению (всех непустых, выпуклых, замкнутых, ограниченных и вьпуклых по переключению) подмножеств из $L^{n}[a, b]$.

Отметим, что понятие выпуклого и выпуклого по переключению множеств суть два независимых понятия.

Далее измеримость множеств понимаем по Лебегу, измеримость многозначных отображений - в смысле [3, гл. $8, \S 8.1]$. Непрерывность многозначных отображений везде понимаем по Хаусдорфу. 


\section{§1. Возмущение выпуклозначного оператора многозначным отображением типа Гаммерштейна с невыпуклыми образами}

Рассмотрим в пространстве $C^{n}[a, b]$ включение

$$
x \in \Psi(x)+V \Phi(x),
$$

где многозначный оператор $\Psi: C^{n}[a, b] \rightarrow \Omega\left(C^{n}[a, b]\right)$ компактен, а многозначное отображение $\Phi: C^{n}[a, b] \rightarrow \Pi\left[L^{n}[a, b]\right]$ обладает следующим свойством: для каждого ограниченного множества $\mathscr{B} \subset C^{n}[a, b]$ образ $\Phi(\mathscr{B})$ имеет равностепенно абсолютно непрерывные интегралы. Линейный непрерьвньй интегральный оператор $V: L^{n}[a, b] \rightarrow C^{n}[a, b]$ определен равенством

$$
(V z)(t)=\int_{a}^{b} V(t, s) z(s) d s, \quad t \in[a, b],
$$

и переводит каждое слабо компактное в $L^{n}[a, b]$ множество в компактное в $C^{n}[a, b]$.

Под решением включения (1) будем понимать такой элемент $x \in C^{n}[a, b]$, для которого справедливо включение (1). Таким образом, каждому решению $x$ включения (1) соответствуют такие $v \in C^{n}[a, b]$ и $z \in L^{n}[a, b]$, что $v \in \Psi(x), z \in \Phi(x)$ и $x=v+V z$. Пусть $H(H(U))$ - множество всех решений (принадлежаших множеству $U)$ включения (1).

Отметим, что многозначные операторы $\Psi$ и $\Phi$, вообще говоря, могут быть и не вольтерровыми операторами. Кроме того, значения $\Phi(x)$ в включении (1) не предполагаются выпуклыми множествами, поэтому образ $V \Phi(x)$ в (1), вообе говоря, не только не является выпуклым, но и замкнутым множеством пространства $C^{n}[a, b]$. И, следовательно, оператор, порожденньй правой частью включения (1), не замкнут.

1.1. Возмущенное включение с ядром оператора $V$, представляющим некоторое множество, содержащее нуль. Здесь предполагается, что ядро оператора $V$, вообше говоря, может состоять не только из нулевого элемента.

Пусть оператор $\mathfrak{M}: C^{n}[a, b] \rightarrow 2^{C^{n}[a, b]}$ определен равенством

$$
\mathfrak{M}(x)=\Psi(x)+V \Phi(x) .
$$

ТЕОРема 1. Пусть $U$ - такое выпуклое, ограниченное, замкнутое множество пространства $C^{n}[a, b]$, что $\mathfrak{M}(U) \subset U . \quad$ И пусть отображсения $\Psi, \Phi$ непрерьвны. Тогда для любого $\varepsilon>0$ и любых функиий $r \in C^{n}[a, b] u$ $w \in L^{n}[a, b]$ существует решение $x \in U$ включения (1) и существуют $v \in \Psi(x)$ $u z \in \Phi(x)$, удовлетворяющие равенству $x=v+V z$, которые обладают следующими свойствами:

$$
\|r-v\|_{C^{n}[a, b]} \leqslant \rho_{C^{n}[a, b]}[r ; \Psi(x)]+\varepsilon ;
$$

для любого измеримого множества $\mathscr{U} \subset[a, b]$ выполняется неравенство

$$
\|w-z\|_{L^{n}(\mathscr{U})} \leqslant \rho_{L^{n}(\mathscr{U})}[w ; \Phi(x)]+\varepsilon \mu(\mathscr{U}) .
$$

Eсли $\Phi: C^{n}[a, b] \rightarrow \Omega\left(\Pi\left[L^{n}[a, b]\right]\right)$, то утверждение справедливо и при $\varepsilon=0$. 
ДоказАТЕльСтво. Так как отображение $\Phi: C^{n}[a, b] \rightarrow \Pi\left[L^{n}[a, b]\right]$ непрерьвно, то согласно [5] для числа $\varepsilon>0$ и функции $w \in L^{n}[a, b]$ существует непрерывное отображение $g: U \rightarrow L^{n}[a, b]$, удовлетворяющее для любого $y \in U$ и любого измеримого множества $\mathscr{U} \subset[a, b]$ включению $g(y) \in \Phi(y)$ и неравенству

$$
\|w-g(y)\|_{L^{n}(\mathscr{U})} \leqslant \rho_{L^{n}(\mathscr{U})}[w ; \Phi(y)]+\varepsilon \mu(\mathscr{U}) .
$$

Далее, рассмотрим отображения $\rho: U \rightarrow \mathbb{R}^{1}$ и $\Psi_{\varepsilon}: U \rightarrow \Omega\left(C^{n}[a, b]\right)$, определенные равенствами

$$
\rho(y)=\rho_{C^{n}[a, b]}[r ; \Psi(y)], \quad \Psi_{\varepsilon}(y)=\overline{\Psi(y) \cap B_{C^{n}[a, b]}[r ; \rho(y)+\varepsilon]} .
$$

В силу непрерывности $\Psi$ отображение $\rho$ непрерьвно, а отображение $\Psi_{\varepsilon}$ полунепрерывно снизу (см. [6, гл. $0, \S 3]$ ). Поэтому согласно теореме Майкла (см. [4]) существует такое непрерывное отображение $f: U \rightarrow C^{n}[a, b]$, что для любого $y \in U$ вьполняется включение $f(y) \in \Psi_{\varepsilon}(y)$. Отсюда согласно определению оператора $\Psi_{\varepsilon}$ отображение $f$ обладает следуюшим свойством: для любого $y \in U$ выполняется неравенство

$$
\|r-f(y)\|_{C^{n}[a, b]} \leqslant \rho_{C^{n}[a, b]}[r ; \Psi(y)]+\varepsilon .
$$

Далее, пусть непрерывный оператор $K: U \rightarrow C^{n}[a, b]$ определен равенством

$$
K(y)=f(y)+V g(y)
$$

Так как согласно условию теоремы справедливы вложения $\mathrm{K}(U) \subset \mathfrak{M}(U) \subset U$, то в силу теоремы Шаудера уравнение

$$
x=f(x)+V g(x)
$$

имеет решение, принадлежашее множеству $U$. Решение уравнения (8) является решением включения (1). Далее, положив в неравенстве (6) $g(x)=z$, а в неравенстве (7) $f(x)=v$ и обозначив $y=x$, получим, что решение $x$ удовлетворяет неравенствам (4), (5).

Докажем вторую часть утверждения теоремы. Пусть отображение $\Phi: C^{n}[a, b]$ $\rightarrow \Omega\left(\Pi\left[L^{n}[a, b]\right]\right)$. Согласно доказанному для каждого $i=1,2, \ldots$ существует решение $x_{i} \in U$ включения (1) и сушествуют $v_{i} \in \Psi\left(x_{i}\right)$ и $z_{i} \in \Phi\left(x_{i}\right)$, удовлетворяющие равенству $x_{i}=v_{i}+V z_{i}$. Причем для функции $v_{i}$ выполняется неравенство (4) при $v=v_{i}$ и $\varepsilon=1 / i$, а для функции $z_{i}$ для любого измеримого множества $\mathscr{U} \subset[a, b]$ выполняется неравенство (5) при $z=z_{i}$ и $\varepsilon=1 / i$. Так как последовательности $\left\{x_{i}\right\},\left\{v_{i}\right\}$ компактны в $C^{n}[a, b]$, а последовательность $\left\{z_{i}\right\}$ слабо компактна в $L^{n}[a, b]$, то, не уменьшая обшности, будем считать, что $x_{i} \rightarrow x, v_{i} \rightarrow v$ в $C^{n}[a, b]$, а $z_{i} \rightarrow z$ слабо в $L^{n}[a, b]$ при $i \rightarrow \infty$. В силу того, что при любом $t \in[a, b]$ $\left(V z_{i}\right)(t) \rightarrow(V z)(t)$ при $i \rightarrow \infty$ (см. [2, гл. VIII, $\S 3$, п. 3.4]), имеем $x=v+V z$. Так как отображение $\Psi$ непрерывно, то $v \in \Psi(x)$. Далее, поскольку $\Phi$ непрерьвно вьпуклозначно и замкнуто в $L^{n}[a, b]$, то $z \in \Phi(x)$ (см. [3, гл. III, §3.1, п. 3.1.2]). Следовательно, $x$ - решение включения (1). 
Покажем, что для решения $x$ выполняются равенства (4), (5) при $\varepsilon=0$. Прежде всего, равенство (4) получается непосредственно предельным переходом. Далее, покажем, что для решения выполняется и неравенство (5) при $\varepsilon=0$. Действительно, так как $z_{i} \rightarrow z$ слабо в $L^{n}[a, b]$, то согласно [3, гл. $3, \S 3.1$, п. 3.1.2] для каждого $m=1,2, \ldots$ найдутся такие числа $i(m), \lambda_{j}^{m} \geqslant 0, j=1,2, \ldots, i(m)$, что $\sum_{j=1}^{i(m)} \lambda_{j}^{m}=1$ и последовательность $\left\{\beta_{m}=\sum_{j=1}^{i(m)} \lambda_{j}^{m} z_{j+m}\right\}$ сходится к $z$ в пространстве $L^{n}[a, b]$. Отсюда получаем

$$
\|w-z\|_{L^{n}[a, b]} \leqslant\left\|z-\beta_{m}\right\|_{L^{n}[a, b]}+\sum_{j=1}^{i(m)} \lambda_{j}^{m}\left\|z_{j+m}-w\right\|_{L^{n}[a, b]} .
$$

В силу выбора последовательности $\left\{z_{i}\right\}$ имеем оценку

$$
\begin{aligned}
\|w-z\|_{L^{n}[a, b]} \leqslant & \left\|z-\beta_{m}\right\|_{L^{n}[a, b]} \\
& +\sum_{j=1}^{i(m)} \lambda_{j}^{m} \rho_{L^{n}[a, b]}\left[w ; \Phi\left(x_{j+m}\right)\right]+(b-a) \sum_{j=1}^{i(m)} \lambda_{j}^{m} \frac{1}{j+m} .
\end{aligned}
$$

Переходя в последнем неравенстве к пределу при $m \rightarrow \infty$, получаем равенство

$$
\|w-z\|_{L^{n}[a, b]}=\rho_{L^{n}[a, b]}[w ; \Phi(x)] .
$$

Из включения $\Phi(x) \in \Omega\left(\Pi\left[L^{n}[a, b]\right]\right)$ вытекает, что последнее равенство вьполняется и для любого измеримого множества $\mathscr{U} \subset[a, b]$. Теорема доказана.

Будем говорить, что для непрерьвного оператора $\mathfrak{A}^{*}: C_{+}^{1}[a, b] \rightarrow C_{+}^{1}[a, b]$ cходятся последовательные приближения, если для любой функции $y_{0} \in C_{+}^{1}[a, b]$, удовлетворяюшей неравенству $y_{0} \leqslant \mathfrak{A}^{*} y_{0}$, последовательные приближения $y_{i+1}=\mathfrak{A}^{*} y_{i}, i=0,1, \ldots$, сходятся в пространстве $C^{1}[a, b]$ к функции $y$, не зависяшей от функции $y_{0}$.

Будем говорить, что отображение $\Psi$ и произведение $V \Phi$ обладают свойством $\left(V \Gamma_{1} ; P_{1}\right)^{\nu}$ на множестве $U \subset C^{n}[a, b]$, если найдутся изотонные непрерывные операторы $\Gamma_{1}: C_{+}^{1}[a, b] \rightarrow L^{1}[a, b]$ и $P_{1}: C_{+}^{1}[a, b] \rightarrow \mathbb{R}^{1}$, удовлетворяющие следующим условиям: для любых $x, y \in U$ и любого измеримого множества $\mathscr{U} \subset[a, b]$ для отображения $\Gamma_{1}$ вьполняется неравенство

$$
h_{L^{n}(\mathscr{U})}[\Phi(x) ; \Phi(y)] \leqslant\left\|\Gamma_{1} Z(x-y)\right\|_{L^{1}(\mathscr{U})} ;
$$

для любых $x, y \in U$ для отображения $P_{1}$ выполняется оценка

$$
h_{C^{n}[a, b]}[\Psi(x) ; \Psi(y)] \leqslant P_{1}(Z(x-y))
$$

для непрерывного оператора $\mathfrak{A}_{1}: C_{+}^{1}[a, b] \rightarrow C_{+}^{1}[a, b]$, определенного равенством

$$
\left(\mathfrak{A}_{1} z\right)(t)=\int_{a}^{b}|V(t, s)|\left(\Gamma_{1} z\right)(s) d s+P_{1}(z)+\nu(t),
$$


сходятся последовательные приближения. Здесь $|V(t, s)|$ - согласованная с пространством $\mathbb{R}^{n}$ норма $(n \times n)$-матрицы $V(t, s)$ в представлении $(2), \nu \in C_{+}^{1}[a, b]$, а отображение $Z: C^{n}[a, b] \rightarrow C_{+}^{1}[a, b]$ определено соотношением

$$
(Z x)(t)=|x(t)|, \quad t \in[a, b] .
$$

Если $U=C^{n}[a, b]$, то в этом случае будем говорить, что отображение $\Psi$ и произведение $V \Phi$ обладают свойством $\left(V \Gamma_{1} ; P_{1}\right)^{\nu}$.

Рассмотрим в пространстве $C^{1}[a, b]$ уравнение

$$
\xi_{\nu}(t)=\int_{a}^{b}|V(t, s)|\left(\Gamma_{1} \xi_{\nu}\right)(s) d s+P_{1}\left(\xi_{\nu}\right)+\nu(t) .
$$

Пусть $q \in C^{n}[a, b]$ и $w_{0} \in L^{n}[a, b]$. Представим функцию $q$ в виде равенства

$$
q=r_{0}+V w_{0}+e
$$

где $r_{0} \in \Psi(q), e=q-r_{0}-V w_{0}$. Пусть функция $\varkappa \in L^{1}[a, b]$ для каждого измеримого $\mathscr{U} \subset[a, b]$ удовлетворяет неравенству

$$
\rho_{L^{n}(\mathscr{U})}\left[w_{0} ; \Phi(q)\right] \leqslant \int_{\mathscr{U}} \varkappa(s) d s,
$$

а функция $\nu_{\varepsilon} \in C_{+}^{1}[a, b]$ для любого $t \in[a, b]$ определена соотношением

$$
\nu_{\varepsilon}(t)=\int_{a}^{b}|V(t, s)|(\varepsilon+\varkappa(s)) d s+\varepsilon+|e(t)|, \quad \varepsilon \geqslant 0,
$$

где $|V(t, s)|$ - согласованная с пространством $\mathbb{R}^{n}$ норма $(n \times n)$-матрицы $V(t, s)$ в представлении $(2), e$ - функция в правой части равенства (14).

Tеорема 2. Пусть отображение $\Psi$ и произведение VФ обладают свойством $\left(V \Gamma_{1} ; P_{1}\right)^{\nu_{\varepsilon}}$ на множестве $U \subset C^{n}[a, b]$, где функция $\nu_{\varepsilon}$ определена равенством (16), $\varepsilon \geqslant 0$, и пусть $q \in U$. Тогда для каждого решения $x \in U$ $(x=v+V z, v \in \Psi(x), z \in \Phi(x))$ включения (1), удовлетворяющего неравенству (4) и при любом измеримом мнохсестве $\mathscr{U} \subset[a, b]$ удовлетворяющего неравенству (5), в которых $r=r_{0}$ и $w=w_{0}$, выполняются следующие оценки: при любом $t \in[a, b]|x(t)-q(t)| \leqslant \xi_{\varepsilon}(t)$;

$$
\left\|v-r_{0}\right\|_{C^{n}[a, b]} \leqslant P_{1}\left(\xi_{\varepsilon}\right)+\varepsilon ;
$$

при почти всех $t \in[a, b]$

$$
\left|z(t)-w_{0}(t)\right| \leqslant \varepsilon+\varkappa(t)+\left(\Gamma_{1} \xi_{\varepsilon}\right)(t)
$$

где $\xi_{\varepsilon}$ - решение уравнения (13) при $\nu=\nu_{\varepsilon}$, для функиий $q, r_{0}, w_{0}$ имеет место представление (14), а $\Gamma_{1}, P_{1}$, и удовлетворяют неравенствам (9), (10), (15), соответственно. 
ДоказАТЕЛЬСтво. Пусть $x(x=v+V z, v \in \Psi(x), z \in \Phi(x))$ - решение включения (1), удовлетворяющее неравенству (4) и при любом измеримом множестве $U \subset[a, b]$ удовлетворяющее неравенству (5), в которых $r=r_{0}, w=w_{0}$ и $\varepsilon \geqslant 0$, где $r_{0}, w_{0}$ - функции в представлении (14). Тогда из неравенства (5) для любого измеримого множества $\mathscr{U} \subset[a, b]$ вытекает соотношение

$$
\left\|z-w_{0}\right\|_{L^{n}(\mathscr{U})} \leqslant \rho_{L^{n}(\mathscr{U})}\left[w_{0} ; \Phi(q)\right]+h_{L^{n}(\mathscr{U})}[\Phi(x) ; \Phi(q)]+\varepsilon \mu(\mathscr{U}) .
$$

Согласно (9) имеем неравенство

$$
h_{L^{n}(\mathscr{U})}[\Phi(x) ; \Phi(q)] \leqslant \int_{\mathscr{U}} \Gamma_{1}(Z(x-q))(s) d s .
$$

Следовательно, из неравенств (15), (19), (20) при любом измеримом множестве $\mathscr{U} \subset[a, b]$ получаем оценку

$$
\left\|z-w_{0}\right\|_{L^{n}(\mathscr{U})} \leqslant \int_{\mathscr{U}}(\varepsilon+\varkappa(s)) d s+\int_{\mathscr{U}}\left(\Gamma_{1} Z(x-q)\right)(s) d s .
$$

Отсюда в силу произвольности множества $\mathscr{U} \subset[a, b]$ при почти всех $t \in[a, b]$ получаем соотношение

$$
\left|z(t)-w_{0}(t)\right| \leqslant \varepsilon+\varkappa(t)+\Gamma_{1}(Z(x-q))(t) .
$$

Из неравенств (4), (10) следует оценка

$$
\left\|v-r_{0}\right\|_{C^{n}[a, b]} \leqslant \rho_{\mathbb{R}^{n}}\left[r_{0} ; \Psi(x)\right]+\varepsilon \leqslant h_{\mathbb{R}^{n}}[\Psi(q) ; \Psi(x)]+\varepsilon \leqslant P_{1}(Z(x-q))+\varepsilon .
$$

Далее, рассмотрим равенство

$$
x(t)-q(t)=v(t)-r_{0}(t)+\int_{a}^{b} V(t, s)\left(z(s)-w_{0}(s)\right) d s-e(t) .
$$

Из представления (23) при любом $t \in[a, b]$ вытекает неравенство

$$
|x(t)-q(t)| \leqslant\left|v(t)-r_{0}(t)\right|+\int_{a}^{b}|V(t, s)|\left|z(s)-w_{0}(s)\right| d s+|e(t)| .
$$

Отсюда согласно оценкам (21), (22) при любом $t \in[a, b]$ имеем соотношение

$$
Z(x-q)(t) \leqslant \int_{a}^{b}|V(t, s)| \Gamma_{1}(Z(x-q))(s) d s+P_{1}(Z(x-q))+\nu_{\varepsilon}(t),
$$

где $\nu_{\varepsilon}, Z$ определены равенствами (16), (12), соответственно. Так как последовательные приближения оператора $\mathfrak{A}_{1}$, определенного равенством (11) при $\nu=\nu_{\varepsilon}$, сходятся, то при любом $t \in[a, b]$ выполняется оценка $|x(t)-q(t)| \leqslant \xi_{\varepsilon}(t)$. В силу последнего неравенства и изотонности отображений $\Gamma_{1}$ и $P_{1}$ из неравенств $(21),(22)$ получаем неравенства (17), (18). Теорема доказана.

Из теорем 1, 2 вытекает 
СЛЕДСТВИЕ 1. Пусть $U$ - такое выпуклое, ограниченное, замкнутое множество пространства $C^{n}[a, b]$, что $\mathfrak{M}(U) \subset U$, әде оператор $\mathfrak{M}$ определен равенством (3). Пусть оператор $\Psi$ и произведение $V \Phi$ обладают свойством $\left(V \Gamma_{1} ; P_{1}\right)^{\nu_{\varepsilon}}$ на множсестве $U$, где функция $\nu_{\varepsilon}$ определена равенством (16), $\varepsilon>0$, и пусть функщия $q$, принадлежсащая $U$, мохет бить представлена равенством (14). Тогда существует решение $x$ включения (1) и существуют $v \in \Psi(x)$ u $z \in \Phi(x)$, удовлетворяющие равенству $x=v+V z$, для которьх при любом $t \in[a, b]$ выполняется неравенство $|x(t)-q(t)| \leqslant \xi_{\varepsilon}(t)$, справедлива оченка (17) и при почти всех $t \in[a, b]$ имеет место соотношение (18), где $\xi_{\varepsilon}$ - решение уравнения (13) при $\nu=\nu_{\varepsilon}$.

Если $\Phi: C^{n}[a, b] \rightarrow \Omega\left(\Pi\left[L^{n}[a, b]\right]\right)$, то утверждение справедливо и при $\varepsilon=0$.

ЗАмечАнИЕ 1. Отметим, что следствие 1 дает несколько больше, чем просто условия существования решения включения (1). Оно дает способ нахождения приближенного решения путем подбора функции $q \in C^{n}[a, b]$. При этом функция $\xi_{\varepsilon}$, зависяшая от функций $q, r_{0} \in C^{n}[a, b]$ и $w_{0} \in L^{n}[a, b]$, дает оценку погрешности приближенного решения (функции $q$ ) включения (1).

Приведем достаточные условия сушествования выпуклого, ограниченного, замкнутого множества $U \subset C^{n}[a, b]$, для которого справедливо вложение $\mathfrak{M}(U) \subset U$.

Пусть отображение $\widetilde{\Psi}:[a, b] \times C^{n}[a, b] \rightarrow \operatorname{comp}\left[\mathbb{R}^{n}\right]$ определено равенством

$$
\widetilde{\Psi}(t, x)=\left\{y \in \mathbb{R}^{n}: \exists z \in \Psi(x), y=z(t)\right\} .
$$

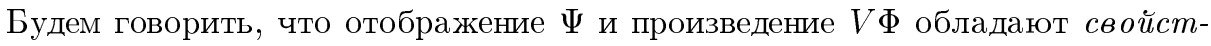
вом $\left(V \Gamma_{2} ; P_{2}\right)$, если найдутся изотонные непрерывные операторы $\Gamma_{2}: C_{+}^{1}[a, b] \rightarrow$ $L^{1}[a, b]$ и $P_{2}: C_{+}^{1}[a, b] \rightarrow C_{+}^{1}[a, b]$, удовлетворяющие следующим условиям: для любого $x \in C^{n}[a, b]$ и любого измеримого множества $\mathscr{U} \subset[a, b]$ выполняется неравенство

$$
\|\Phi(x)\|_{L^{n}(\mathcal{U})} \leqslant\left\|\Gamma_{2} Z(x)\right\|_{L^{1}(\mathcal{U})}
$$

для любого $t \in[a, b]$ и любого $x \in C^{n}[a, b]$ вьполняется оценка

$$
\|\widetilde{\Psi}(t, x)\|_{\mathbb{R}^{n}} \leqslant P_{2}(Z(x))(t)
$$

для непрерывного оператора $\mathfrak{A}_{2}: C_{+}^{1}[a, b] \rightarrow C_{+}^{1}[a, b]$, определенного равенством

$$
\left(\mathfrak{A}_{2} z\right)(t)=\int_{a}^{b}|V(t, s)|\left(\Gamma_{2} z\right)(s) d s+P_{2}(z)(t),
$$

сходятся последовательные приближения.

Здесь $|V(t, s)|$ - согласованная с пространством $\mathbb{R}^{n}$ норма $(n \times n)$-матрицы $V(t, s)$ в представлении (2), отображения $Z$ и $\widetilde{\Psi}$ определены равенствами (12) и $(25)$, соответственно.

Пусть $\omega \in C^{1}[a, b]$ - неподвижная точка оператора $\mathfrak{A}_{2}$, определенного равенством (28), и пусть непрерьвное отображение $W: C^{n}[a, b] \rightarrow C^{n}[a, b]$ задано соотношением

$$
(W x)(t)= \begin{cases}x(t), & \text { если }|x(t)| \leqslant \omega(t), \\ \frac{x(t)}{|x(t)|} \omega(t), & \text { если }|x(t)|>\omega(t) .\end{cases}
$$


ЛЕмма 1. Пусть оператор $\Psi$ и произведение $V \Phi$ обладают свойством $\left(V \Gamma_{2} ; P_{2}\right)$. Тогда множество $U=\overline{\mathrm{Co}} \mathfrak{M}\left(W\left(C^{n}[a, b]\right)\right)$ - выпуклый компакт пространства $C^{n}[a, b]$, для которого справедливо вложение $\mathfrak{M}(U) \subset U$, операторы $W$ и $\mathfrak{M}$ определены равенствами (29) и (3), соответственно.

ДокАЗАТЕЛЬСтво. Прежде всего, согласно определению оператора $W$ множество $W\left(C^{n}[a, b]\right)$ ограничено, поэтому множество $U$ - выпуклый компакт в $C^{n}[a, b]$. Далее, пусть $y \in \operatorname{co} \mathfrak{M}\left(W\left(C^{n}[a, b]\right)\right)$. Тогда найдутся такие числа $\lambda_{i} \geqslant 0, i=1,2, \ldots, m$, что $\sum_{i=1}^{m} \lambda_{i}=1$, и элементы $z_{i} \in \mathfrak{M}\left(W\left(C^{n}[a, b]\right)\right)$, $i=1,2, \ldots, m$, для которых справедливо равенство

$$
y=\sum_{i=1}^{m} \lambda_{i} z_{i} .
$$

Согласно определению оператора $\mathfrak{M}$ для каждого $z_{i}, i=1,2, \ldots, m$, найдется $p_{i} \in$ $C^{n}[a, b]$, что $z_{i} \in \Psi\left(W\left(p_{i}\right)\right)+V \Phi\left(W\left(p_{i}\right)\right)$. Поэтому для каждого $z_{i}, i=1,2, \ldots, m$, найдутся $z_{i}^{1} \in \Psi\left(W\left(p_{i}\right)\right)$ и $z_{i}^{2} \in \Phi\left(W\left(p_{i}\right)\right)$, что

$$
z_{i}=z_{i}^{1}+V z_{i}^{2}
$$

Следовательно, согласно свойствам $\left(V \Gamma_{2} ; P_{2}\right)$ для каждого $t \in[a, b]$ справедливы оценки

$$
\begin{gathered}
\left|z_{i}^{1}(t)\right| \leqslant P_{2}\left(Z W\left(p_{i}\right)\right)(t) \leqslant P_{2}(\omega)(t) \\
\left|\left(V z_{i}^{2}\right)(t)\right| \leqslant \int_{a}^{b}|V(t, s)| \Gamma_{2}\left(Z W\left(p_{i}\right)\right)(s) d s \leqslant \int_{a}^{b}|V(t, s)|\left(\Gamma_{2} \omega\right)(s) d s .
\end{gathered}
$$

Из равенства (31) и полученных оценок для любого $t \in[a, b]$ вытекает соотношение

$$
\left|z_{i}(t)\right| \leqslant \int_{a}^{b}|V(t, s)|\left(\Gamma_{2} \omega\right)(s) d s+P_{2}(\omega)(t)=\left(\mathfrak{A}_{2} \omega\right)(t)=\omega(t)
$$

так как $\omega$ - неподвижная точка оператора $\mathfrak{A}_{2}$, определенного равенством (28). Из равенства (30) и оценки (32) для любого $t \in[a, b]$ получаем соотношение $|y(t)| \leqslant \omega(t)$. Поэтому для любого $y \in \overline{\operatorname{co}} \mathfrak{M}\left(W\left(C^{n}[a, b]\right)\right)=U$ и для любого $t \in[a, b]$ выполняется неравенство $|y(t)| \leqslant \omega(t)$. Отсюда и из определения оператора $W$ (см. (29)) следует вложение $U \subset W\left(C^{n}[a, b]\right)$. Поэтому $\mathfrak{M}(U) \subset U$. Лемма доказана.

Таким образом, из леммы 1 и следствия 1 вытекает

СлЕДСТВИЕ 2. Пусть оператор $\Psi$ и произведение $V \Phi$ обладают свойствами $\left(V \Gamma_{1} ; P_{1}\right)^{\nu_{\varepsilon}}$ и $\left(V \Gamma_{2} ; P_{2}\right)$, әде функиия $\nu_{\varepsilon}$ определена равенством (16), $\varepsilon>0$, и пусть функция $q \in C^{n}[a, b]$ может быть представлена равенством (14). Тогда существует решение $x$ включения (1) и существуют $v \in \Psi(x)$ u $z \in \Phi(x)$, удовлетворяющие равенству $x=v+V z$, для которых при любом 
$t \in[a, b]$ выполняется неравенство $|x(t)-q(t)| \leqslant \xi_{\varepsilon}(t)$, справедлива оценка (17) и при почти всех $t \in[a, b]$ имеет место соотношение (18), где $\xi_{\varepsilon}-$ решение уравнения (13) при $\nu=\nu_{\varepsilon}$.

Если $\Phi: C^{n}[a, b] \rightarrow \Omega\left(\Pi\left[L^{n}[a, b]\right]\right)$, то утверждение справедливо и при $\varepsilon=0$.

Будем говорить, что функция $x \in C^{n}[a, b]$ является квазирешением включения (1), если сушествует $v \in \Psi(x)$ и последовательность $w_{i} \in \Phi(x), i=1,2, \ldots$, такие, что $y_{i}=v+V w_{i} \rightarrow x$ в $C^{n}[a, b]$ при $i \rightarrow \infty$. Пусть $\mathscr{H}-$ множество всех квазирешений включения (1).

Определим отображение $\operatorname{co} \Phi: C^{n}[a, b] \rightarrow \Omega\left(L^{n}[a, b]\right)$ равенством

$$
(\operatorname{co} \Phi)(x)=\overline{\operatorname{co}}(\Phi(x))
$$

Рассмотрим включение

$$
x \in \Psi(x)+V \operatorname{co} \Phi(x) .
$$

Пусть $H_{\text {со }}\left(H_{\text {со }}(U)\right)$ - множество всех решений (принадлежащих множеству $U$ ) включения (33).

Теорема 3. Справедливо следующее: $H_{\text {со }}=\mathscr{H}$.

ДоказАтельство. Сначала докажем, что $H_{\text {со }} \subset \mathscr{H}$. Пусть $x \in H_{\text {со }}$. Тогда найдутся функции $v \in \Psi(x)$ и $w \in \operatorname{co} \Phi(x)$ такие, что выполняется равенство $x=v+V w$. Согласно [1] для функции $w$ найдется такая последовательность $w_{i} \in \Phi(x), i=1,2, \ldots$, что $w_{i} \rightarrow w$ слабо в $L^{n}[a, b]$ при $i \rightarrow \infty$. Это означает, что при любом $t \in[a, b]$

$$
y_{i}(t)=v(t)+\left(V w_{i}\right)(t) \rightarrow x(t)=v(t)+(V w)(t)
$$

(см. [2, гл. VIII, $\S 3$, п. 3.4]). Так как последовательность $\left\{V w_{i}\right\}$ компактна в $C^{n}[a, b]$, то можно считать, что $y_{i} \rightarrow x$ в $C^{n}[a, b]$ при $i \rightarrow \infty$. Следовательно, $H_{\text {со }} \subset \mathscr{H}$.

Докажем теперь вложение $\mathscr{H} \subset H_{\text {со }}$. Пусть $x \in \mathscr{H}$. Тогда найдется $v \in \Psi(x)$ и последовательность $w_{i} \in \Phi(x)$ такие, что $y_{i}=v+V w_{i} \rightarrow x$ в $C^{n}[a, b]$ при $i \rightarrow \infty$. Поскольку последовательность $\left\{w_{i}\right\}$ слабо компактна в $L^{n}[a, b]$, то, не уменьшая обшности, можно считать, что $w_{i} \rightarrow w$ слабо в $L^{n}[a, b]$ при $i \rightarrow \infty$ для некоторого $w \in L^{n}[a, b]$. Так как $w_{i} \in \operatorname{co} \Phi(x)$, то $w \in \operatorname{co} \Phi(x)$ (см. [3, гл. III, §3.1, п. 3.1.2]) и $x=v+V w$, т.е. $x \in H_{\text {со }}$. Следовательно, $\mathscr{H} \subset H_{\text {со }}$. Теорема доказана.

ЗАмечаниЕ 2. Отметим, что теорема 3 справедлива без предположения какой-либо непрерывности операторов $\Psi$ и $\Phi$.

Будем говорить, что отображение $\Phi: C^{n}[a, b] \rightarrow \Pi\left[L^{n}[a, b]\right]$ ослабленно замкнуто в $x \in C^{n}[a, b]$, если для любой последовательности $x_{i} \rightarrow x$ в $C^{n}[a, b]$ при $i \rightarrow \infty$ и для любой последовательности $y_{i} \in \Phi\left(x_{i}\right), i=1,2, \ldots$, удовлетворяющей условию $y_{i} \rightarrow y$ слабо в $L^{n}[a, b]$ при $i \rightarrow \infty$, выполняется включение $y \in \Phi(x)$. Если отображение $\Phi$ ослабленно замкнуто в каждой точке множества $U$, то будем говорить, что отображение $\Phi$ ослабленно замкнуто на $U$. 
Лемма 2. Если отображсение со $\Phi: C^{n}[a, b] \rightarrow \Omega\left(L^{n}[a, b]\right)$ полунепрерывно сверху на множестве $U \subset C^{n}[a, b]$, то отображсение $\operatorname{co} \Phi$ ослабленно замкнуто на $U$.

ДокаЗАТЕльСтво. Действительно, пусть $x_{i} \in U, x_{i} \rightarrow x$ в $C^{n}[a, b], i \rightarrow \infty$, и последовательность $y_{i} \in \operatorname{co} \Phi\left(x_{i}\right), i=1,2, \ldots$, при $i \rightarrow \infty$ слабо сходится в пространстве $L^{n}[a, b]$ к $y$. Пусть $\widetilde{y}_{i} \in \operatorname{co} \Phi(x), i=1,2, \ldots$, удовлетворяет равенству

$$
\left\|y_{i}-\widetilde{y}_{i}\right\|_{L^{n}[a, b]}=\rho_{L^{n}[a, b]}\left[y_{i} ; \operatorname{co} \Phi(x)\right] .
$$

Согласно полунепрерывности сверху отображения $\operatorname{co} \Phi y_{i}-\widetilde{y}_{i} \rightarrow 0$ в $L^{n}[a, b]$ при $i \rightarrow \infty$. Так как множество $\overline{\mathrm{co}}(\Phi(x))$ замкнуто в слабой топологии пространства $L^{n}[a, b]$ (см. [3, гл. III, §3.1, п. 3.1.2]), то $y \in \operatorname{co} \Phi(x)$.

Из леммы 2 вытекает

Теорема 4. Пусть $U$ - такое выпуклое, ограниченное, замкнутое множество пространства $C^{n}[a, b]$, что $\mathfrak{M}(U) \subset U$, где оператор $\mathfrak{M}$ определен равенством (3). И пусть отображения $\Psi$, соФ полунепрерывны сверху или снизу на $U$. Тогда $\mathscr{H} \neq \varnothing$.

ДокАЗАТЕЛЬСТво. Пусть многозначное отображение

$$
\mathfrak{M}_{\mathrm{co}}: C^{n}[a, b] \rightarrow \Omega\left(C^{n}[a, b]\right)
$$

определено равенством

$$
\mathfrak{M}_{\text {со }}(x)=\Psi(x)+V \operatorname{co} \Phi(x) .
$$

Покажем, что $\mathfrak{M}_{\text {со }}(U) \subset U$. Действительно, пусть $x \in U$ и $z \in \Psi(x)+V \operatorname{co} \Phi(x)$. Тогда найдутся такие $z_{1} \in \Psi(x)$ и $z_{2} \in \operatorname{co} \Phi(x)$, что выполняется равенство

$$
z=z_{1}+V z_{2}
$$

Так как $z_{2} \in \operatorname{co} \Phi(x)$, то найдутся числа $\lambda_{i} \geqslant 0, i=1,2, \ldots, m$, удовлетворяющие равенству $\sum_{i=1}^{m} \lambda_{i}=1$, и элементы $z_{2}^{i} \in \Phi(x), i=1,2, \ldots, m$, для которых справедливо соотношение

$$
z_{2}=\sum_{i=1}^{m} \lambda_{i} z_{2}^{i} .
$$

Так как $z_{1}+z_{2}^{i} \in \mathfrak{M}(x), i=1,2, \ldots, m$, то $z_{1}+z_{2}^{i} \in U, i=1,2, \ldots, m$. Поэтому из выпуклости множества $U$ и равенств $(35),(36)$ следует, что $z \in U$. Отсюда согласно непрерывности оператора $V$ и замкнутости множества $U$ для любого $x \in U$ получаем вложение $\mathfrak{M}_{\text {со }}(x) \subset U$, т.е. $\mathfrak{M}_{\text {со }}(U) \subset U$.

Далее, пусть отображения $\Psi$ и со $\Phi$ полунепрерывны сверху на $U$. Тогда согласно лемме 2 оператор $\mathfrak{M}_{\text {со }}$, определенньй равенством (34), замкнут на $U$. Поэтому согласно доказанному вложению $\mathfrak{M}_{\text {со }}(U) \subset U$ и теореме Какутани (см. $[2$, гл. XVI, § 5]) включение (33) имеет решение. Поэтому в силу теоремы $3 \mathscr{H} \neq \varnothing$.

Пусть теперь отображение $\Psi$ полунепрерывно снизу на $U$, а отображение полунепрерывно сверху. Тогда согласно теореме Майкла (см. [4]) сушествует непрерывное отображение $f: U \rightarrow C^{n}[a, b]$, для любого $x \in U$ удовлетворяюшее 
условию $f(x) \in \Psi(x)$. Рассмотрим отображение $\widetilde{\mathfrak{M}}: U \rightarrow \Omega\left(C^{n}[a, b]\right)$, определенное равенством

$$
\widetilde{\mathfrak{M}}(x)=f(x)+V \operatorname{co} \Phi(x) .
$$

Согласно лемме 2 оператор $\widetilde{\mathfrak{M}}$ замкнут, поэтому опять согласно теореме Какутани и теореме 3 множество $\mathscr{H} \neq \varnothing$.

Аналогично рассматривается случай, когда отображение $\Psi$ полунепрерывно сверху, а отображение соФ полунепрерывно снизу или полунепрерьвно сверху. Теорема доказана.

Из теоремы 4 и леммы 1 вытекает

СЛЕДСТВИЕ 3. Пусть оператор $\Psi$ и произведение VФ обладают свойством $\left(V \Gamma_{2} ; P_{2}\right)$, и отображения $\Psi, \operatorname{co} \Phi$ полунепрерьвны снизу или сверху на $C^{n}[a, b]$. Тогда $\mathscr{H} \neq \varnothing$.

Пусть отображение $F:[a, b] \times C^{n}[a, b] \rightarrow \operatorname{comp}\left[\mathbb{R}^{n}\right]$ обладает следующим свойством: при каждом фиксированном $x \in C^{n}[a, b]$ отображение $F(\cdot, x)$ измеримо и удовлетворяет равенству

$$
\Phi(x)=\left\{y \in L^{n}[a, b]: y(t) \in F(t, x) \text { при п.в. } t \in[a, b]\right\} .
$$

Такое отображение существует (см. [1]). Далее, отображение ext $\Phi: C^{n}[a, b] \rightarrow$ $\Pi\left[L^{n}[a, b]\right]$ определено равенством

$$
(\operatorname{ext} \Phi)(x)=\left\{y \in L^{n}[a, b]: y(t) \in \overline{\operatorname{ext}}(\overline{c o} F(t, x)) \text { при п.в. } t \in[a, b]\right\} .
$$

Отметим, что согласно [1] при каждом $x \in C^{n}[a, b]$ множество $(\operatorname{ext} \Phi)(x)$, определенное равенством (37), - минимальное по включению, выпуклое по переключению, замкнутое в пространстве $L^{n}[a, b]$ множество, содержашееся в множестве $\Phi(x)$ и удовлетворяюшее условию

$$
\overline{\mathrm{CO}}((\operatorname{ext} \Phi)(x))=\overline{\mathrm{CO}}(\Phi(x)) .
$$

Рассмотрим в пространстве $C^{n}[a, b]$ включение

$$
x \in \Psi(x)+V(\operatorname{ext} \Phi)(x)
$$

Пусть $\mathscr{H}_{\text {ext }}$ - множество всех квазирешений включения (39) и пусть $H_{\text {ext }}\left(H_{\text {ext }}(U)\right)$ - множество всех решений (принадлежаших множеству $U$ ) включения (39).

Из равенства (38), теорем 3,4 вытекают

СледСтвие 4. Справедливо следующее: $H_{\text {со }}=\mathscr{H}_{\text {ext }}$.

СЛЕДСТВИЕ 5. Пусть $U$ - такое выпуклое, ограниченное, замкнутое множество пространства $C^{n}[a, b]$, что $\mathfrak{M}(U) \subset U$, где оператор $\mathfrak{M}$ определен равенством (3). И пусть отображения $\Psi, \operatorname{co} \Phi$ полунепрерывны сверху или снизу на $U$. Тогда $\mathscr{H}_{\mathrm{ext}} \neq \varnothing$. 
СлЕДСТВИЕ 6. Пусть оператор $\Psi$ и произведение VФ обладают свойством $\left(V \Gamma_{2} ; P_{2}\right)$, и пусть отображения $\Psi, \operatorname{co} \Phi$ полунепрерьвны снизу или сверху на $U$. Тогда $\mathscr{H}_{\text {ext }} \neq \varnothing$.

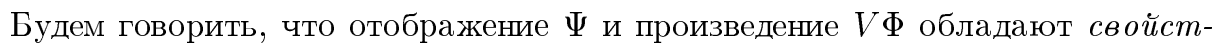
вом А на множестве $U \subset C^{n}[a, b]$, если для любого $\varepsilon>0$ сушествует $\delta>0$ такое, что для любого $\nu \in C_{+}^{1}[a, b]$, удовлетворяюшего неравенству $\|\nu\|_{C^{1}[a, b]} \leqslant \delta$, вьполняется свойство $\left(V \Gamma_{1}, P_{1}\right)^{\nu}$ на множестве $U$, в котором для отображений $\Gamma_{1}$ и $P_{1}$ справедливы равенства $\Gamma_{1}(0)=0, P_{1}(0)=0$, а решение уравнения (13) удовлетворяет неравенству $\left\|\xi_{\nu}\right\|_{C^{1}[a, b]}<\varepsilon$. Если $U=C^{n}[a, b]$, то в этом случае будем говорить, что отображение $\Psi$ и произведение $V \Phi$ обладают свойством А.

Tеорема 5. Пусть $U$ - такое выпуклое, ограниченное, замкнутое множество пространства $C^{n}[a, b]$, что $\mathfrak{M}(U) \subset U$, әде отображение $\mathfrak{M}$ определено равенством (3). Далее, пусть оператор $\Psi$ и произведение $V \Phi$ обладают свойством А на множестве $U$. Тогда $\overline{H(U)}=H_{\mathrm{co}}(U)$, где $\overline{H(U)}-$ замыкание множества $H(U)$ в пространстве $C^{n}[a, b]$.

ДокАЗАТЕЛЬСТво. Так как $\Phi(x) \subset \operatorname{co} \Phi(x)$, то $H(U) \subset H_{\text {со }}(U)$. Поскольку $H_{\text {со }}(U)$ - замкнутое множество пространства $C^{n}[a, b]$, то остается показать, что $H_{\text {co }} \subset \overline{H(U)}$.

Пусть $x \in H_{\text {со }}(U)$. Согласно теореме $3 x$-квазирешение включения (1). Поэтому для $x$ найдется $v \in \Psi(x)$ и последовательность $w_{i} \in \Phi(x), i=1,2, \ldots$, такие, что последовательность $y_{i}=v+V w_{i} \rightarrow x$ в $C^{n}[a, b]$ при $i \rightarrow \infty$.

Пусть для каждого $i=1,2, \ldots$ функция $\overline{v_{i}} \in \Psi\left(y_{i}\right)$ определена равенством

$$
\left\|v-\overline{v_{i}}\right\|_{C^{n}[a, b]}=\rho_{C^{n}[a, b]}\left[v ; \Psi\left(y_{i}\right)\right] .
$$

Очевидно, что $\overline{v_{i}} \rightarrow v$ в $C^{n}[a, b]$ при $i \rightarrow \infty$. Для каждого $i=1,2, \ldots$ запишем $y_{i}$ в виде

$$
y_{i}=\overline{v_{i}}+V w_{i}+e_{i},
$$

где $e_{i}=v-\overline{v_{i}}$. Так как отображение $\Psi$ и произведение $V \Phi$ обладают свойством А на множестве $U$ и так как $y_{i} \in U, i=1,2, \ldots(\mathfrak{M}(U) \subset U)$, то для любого измеримого множества $\mathscr{U} \in[a, b]$ выполняется оценка

$$
\rho_{L^{n}(\mathscr{U})}\left[w_{i} ; \Phi\left(y_{i}\right)\right] \leqslant h_{L^{n}(\mathscr{U})}\left[\Phi(x) ; \Phi\left(y_{i}\right)\right] \leqslant \int_{\mathscr{U}}\left(\Gamma_{1}\left(Z\left(x-y_{i}\right)\right)\right)(s) d s,
$$

где $Z$ определено равенством (12). Из неравенства (41) и следствия 1 вытекает, что для каждой функции $y_{i}, i=1,2, \ldots$, которая может быть представлена в виде (40), найдется решение включения (1) $x_{i} \in U$, для которого при любом $t \in[a, b]$ выполняется неравенство

$$
\left|x_{i}(t)-y_{i}(t)\right| \leqslant \xi_{i}(t)
$$

где $\xi_{i}$ - решение уравнения (13) с функцией $\nu=\nu_{i}$, при любом $t \in[a, b]$ определенной равенством

$$
\nu_{i}(t)=\int_{a}^{b}|V(t, s)|\left(\frac{1}{i}+\Gamma_{1}\left(Z\left(x-y_{i}\right)\right)(s)\right) d s+\frac{1}{i}+\left|e_{i}(t)\right|,
$$


где $e_{i}=v-\overline{v_{i}}$. Так как отображение $\Gamma_{1}$ непрерывно и удовлетворяет равенству $\Gamma_{1}(0)=0$, то $\nu_{i} \rightarrow 0$ в $C^{1}[a, b]$ при $i \rightarrow \infty$. Отсюда и из определения свойства А вытекает, что $\xi_{i} \rightarrow 0$ в $C^{1}[a, b]$ при $i \rightarrow \infty$. Следовательно, из неравенства (42) получаем, что $x_{i}-y_{i} \rightarrow 0$ в $C^{n}[a, b]$ при $i \rightarrow \infty$. Поэтому $x_{i} \rightarrow x$ в $C^{n}[a, b]$ при $i \rightarrow \infty$. Таким образом, $H_{\mathrm{co}}(U) \subset \overline{H(U)}$. Теорема доказана.

Из леммы 1 и теоремы 4 вытекает

СЛЕДСТВИЕ 7. Пусть оператор $\Psi$ и произведение $V \Phi$ обладают свойствами А $и\left(V \Gamma_{2} ; P_{2}\right)$. Тогда $\bar{H}=H_{\mathrm{co}}$, где $\bar{H}$ - замыкание множества $Н$ в пространстве $C^{n}[a, b]$.

1.2. Возмущенное включение с разложимым множеством решений и ядром оператора $V$, состоящим только из нулевого элемента. Здесь предполагается, что ядро оператора $V$ состоит только из нулевого элемента, а также

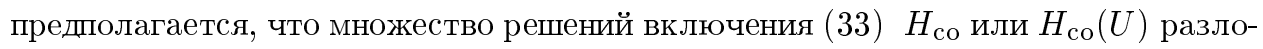
жимы (определение см. ниже). В этом случае удается установить более тонкое утверждение, чем теорема 5.

Будем говорить, что множество $H_{\mathrm{co}}\left(H_{\mathrm{co}}(U)\right)$ разложимо по многозначным отображсениям $\Psi, \operatorname{co} \Phi$ или просто разложимо, если каждое решение $x \in H_{\text {со }}$ $\left(H_{\mathrm{co}}(U)\right)$ однозначно представимо в виде

$$
x=v+V z,
$$

где $v \in \Psi(x), z \in(\operatorname{co} \Phi)(x)$.

Далее, определим отображение $\sigma: H_{\text {со }} \rightarrow C^{n}[a, b]$ равенством $\sigma(x)=v$, где функция $v \in C^{n}[a, b]$ определена представлением (43). Обозначим через $V^{-1}(y)$ прообраз элемента $y \in C^{n}[a, b]$ оператора $V$. Зафиксируем некоторое отображение $g: H_{\text {со }}(U) \rightarrow L^{n}[a, b]$ и рассмотрим функционал $\eta: H_{\text {со }}(U) \rightarrow \mathbb{R}^{1}$, определенный соотношением

$$
\eta(x)=\eta(x, g(x))=\left\|V^{-1}(x-\sigma(x))-g(x)\right\|_{L^{n}[a, b]} .
$$

Отметим, что если $x \in H_{\text {со }}(U)$, то прообраз $V^{-1}(x-\sigma(x)) \neq \varnothing$ и состоит из единственного элемента в силу условия $V^{-1}(0)=0$.

Лемма 3. Пусть $U \subset C^{n}[a, b]$ - ограниченное, замкнутое мнохество, и пусть многозначные отображсения $\Psi: C^{n}[a, b] \rightarrow \Omega\left(C^{n}[a, b]\right), \operatorname{co} \Phi: C^{n}[a, b] \rightarrow$ $\Omega\left(\Pi\left[L^{n}[a, b]\right]\right)$ полунепрерьвны сверху на $U$, а оператор $g: H_{\mathrm{co}}(U) \rightarrow L^{n}[a, b]$ непрерывен. Тогда функционал $\eta$ полунепрерывен снизу на $H_{\mathrm{co}}(U)$.

ДокАЗАТЕльство. Покажем, что множество $\left\{x \in H_{\text {со }}(U): \eta(x) \leqslant \tau\right\}$ замкнуто для любого $\tau \geqslant 0$. Прежде всего, в силу леммы 2 и замкнутости отображения $\Psi$ множество $H_{\text {со }}(U)$ замкнуто. Далее, пусть последовательность $\left\{x_{i}\right\} \subset H_{\text {со }}(U)$ такова, что $x_{i} \rightarrow x \in H_{\text {со }}(U)$ в $C^{n}[a, b]$ при $i \rightarrow \infty$ и $\eta\left(x_{i}\right) \leqslant \tau$ для всех $i=1,2, \ldots$ Обозначим $z_{i}=V^{-1}\left(x_{i}-\sigma\left(x_{i}\right)\right) \in \operatorname{co} \Phi\left(x_{i}\right), i=1,2, \ldots$ Не уменьшая общности, можно считать, что последовательность $\left\{z_{i}\right\}$ сходится слабо в $L^{n}[a, b]$ к некоторому элементу $z_{0} \in L^{n}[a, b]$. Покажем, что $z_{0}=V^{-1}(x-\sigma(x))$. Пусть $\sigma\left(x_{i}\right) \rightarrow v$ в $C^{n}[a, b]$ при $i \rightarrow \infty$. Так как для любого $i=1,2, \ldots$ справедливо равенство 
$x_{i}=\sigma\left(x_{i}\right)+V z_{i}$, то, переходя в этом равенстве к пределу и учитывая, что при любом $t \in[a, b]\left(V z_{i}\right)(t) \rightarrow\left(V z_{0}\right)(t)$ при $i \rightarrow \infty$ (см. [2, гл. VIII, §3, п. 3.4]), получаем соотношение $x=v+V z_{0}$, где $v \in \Psi(x), z_{0} \in \operatorname{co} \Phi(x)$ (см. лемму 2). Отсюда в силу определения отображения $\sigma$ справедливо равенство $x=\sigma(x)+V z_{0}$, т.е. $z_{0}=V^{-1}(x-\sigma(x))$.

Для любого $i=1,2, \ldots$ введем функцию $\alpha_{i} \in L^{1}[a, b]$, определенную равенством $\alpha_{i}(t)=\left|z_{i}(t)-g\left(x_{i}\right)(t)\right|$. Так как последовательность $\left\{z_{i}\right\}$ сходится слабо в $L^{n}[a, b]$ к $z_{0}$ при $i \rightarrow \infty$, а отображение $g$ непрерывно, то последовательность $\left\{\alpha_{i}\right\}$ слабо компактна. Поэтому, не уменьшая общности, можно считать, что последовательность $\alpha_{i}$ сходится слабо к некоторому элементу $\alpha \in L^{1}[a, b]$. Тогда согласно [3, гл. III, $\S 3.1$, п. 3.1.2] для каждого $m=1,2, \ldots$ найдутся такие числа $i(m), \lambda_{j}^{m} \geqslant 0, j=1,2, \ldots, i(m)$, что $\sum_{j=1}^{i(m)} \lambda_{j}^{m}=1$ и последовательность $\left\{\beta_{m}=\sum_{j=1}^{i(m)} \lambda_{j}^{m} \alpha_{j+m}\right\}$ сходится к $\alpha$ в пространстве $L^{n}[a, b]$. Ниже, не уменьшая общности, будем считать, что $\beta_{m} \rightarrow \alpha, g\left(x_{m}\right) \rightarrow g(x)$ почти всюду на $[a, b]$ при $m \rightarrow \infty$. Для любого $m=1,2, \ldots$ функции $y_{m}, p_{m} \in L^{n}[a, b]$ определим равенствами

$$
y_{m}(t)=\sum_{j=1}^{i(m)} \lambda_{j}^{m} z_{j+m}(t), \quad p_{m}(t)=\sum_{j=1}^{i(m)} \lambda_{j}^{m} g\left(x_{j+m}\right)(t) .
$$

Из определений функций $y_{m}, p_{m}$ при почти всех $t \in[a, b]$ получаем оценку

$$
\left|y_{m}(t)-p_{m}(t)\right| \leqslant \sum_{j=1}^{i(m)} \lambda_{j}^{m}\left|z_{j+m}(t)-g\left(x_{j+m}\right)(t)\right|=\beta_{m}(t)
$$

Таким образом, из неравенства (46) при почти всех $t \in[a, b]$ следует включение

$$
y_{m}(t) \in \overline{B_{\mathbb{R}^{n}}\left[p_{m}(t), \beta_{m}(t)\right]}
$$

Из второго равенства (45) вытекает, что $p_{m} \rightarrow g(x)$ почти всюду на $[a, b]$ при $m \rightarrow \infty$. Поэтому по построению при почти всех $t \in[a, b]$ выполняется равенство

$$
\lim _{m \rightarrow \infty} h_{\mathbb{R}^{n}}\left[\overline{B_{\mathbb{R}^{n}}\left[p_{m}(t), \beta_{m}(t)\right]} ; \overline{B_{\mathbb{R}^{n}}[g(x)(t), \alpha(t)]}\right]=0 .
$$

Пусть для каждого $m=1,2, \ldots$ функции $\widetilde{y}_{m} \in L^{n}[a, b]$ при почти всех $t \in[a, b]$ удовлетворяют включению $\widetilde{y}_{m}(t) \in \overline{B_{\mathbb{R}^{n}}[g(x)(t), \alpha(t)]}$ и соотношению

$$
\left|\widetilde{y}_{m}(t)-y_{m}(t)\right|=\rho_{\mathbb{R}^{n}}\left[y_{m}(t) ; \overline{B_{\mathbb{R}^{n}}[g(x)(t) ; \alpha(t)]}\right] .
$$

Из определения функций $\widetilde{y}_{m}$ и равенства (48) следует, что при почти всех $t \in[a, b]$ $\left|\widetilde{y}_{m}(t)-y_{m}(t)\right| \rightarrow 0$ при $m \rightarrow \infty$. Так как из первого равенства (45) вытекает, что $y_{m} \rightarrow z_{0}$ слабо в $L^{n}[a, b]$ при $m \rightarrow \infty$, то и $\widetilde{y}_{m} \rightarrow z_{0}$ слабо в $L^{n}[a, b]$ при $m \rightarrow \infty$. А так как множество $\overline{B_{\mathbb{R}^{n}}[g(x)(t), \alpha(t)]}$ выпукло, то при почти всех $t \in[a, b]$ справедливо включение $z_{0}(t) \in \overline{B_{\mathbb{R}^{n}}[g(x)(t), \alpha(t)]}$, т.е. для $z_{0}$ при почти всех $t \in[a, b]$ 
вьполняется оценка $\left|z_{0}(t)-g(x)(t)\right| \leqslant \alpha(t)$. Отсюда согласно определению функций $\alpha_{i}, i=1,2, \ldots$, получаем соотношение

$$
\int_{a}^{b}\left|z_{0}(t)-g(x)(t)\right| d t \leqslant \int_{a}^{b} \alpha(t) d t=\lim _{i \rightarrow \infty} \int_{a}^{b} \alpha_{i}(t) d t \leqslant \tau .
$$

Следовательно, $\eta(x) \leqslant \tau$, а это означает, что множество $\left\{x \in H_{\text {со }}(U): \eta(x) \leqslant \tau\right\}$ замкнуто и поэтому функционал $\eta$ полунепрерывен снизу. Лемма доказана.

Рассмотрим функционал $\Theta: H_{\text {со }}(U) \rightarrow \mathbb{R}^{1}$, заданный равенством

$$
\Theta(x)=\Theta(x, g(x))=\rho_{L^{n}[a, b]}[g(x) ;(\operatorname{ext} \Phi)(x)] .
$$

Теорема 6. Пусть $U$ - такое выпуклое, ограниченное, замкнутое множество пространства $C^{n}[a, b]$, что $\mathfrak{M}(U) \subset U$, где оператор $\mathfrak{M}$ определен равенством (3). И пусть отображсение $\Psi$ и произведение $V \operatorname{co} \Phi$ обладают свойством А на множестве $U$. Тогда $H_{\mathrm{ext}}(U) \neq \varnothing$ и $\overline{H_{\mathrm{ext}}(U)}=H_{\mathrm{co}}(U)$, где $\overline{H_{\text {ext }}(U)}$ - замыкание множества $H_{\text {ext }}(U)$ в пространстве $C^{n}[a, b]$.

ДокаЗАтельство. Докажем, что $\overline{H_{\text {ext }}(U)}=H_{\text {со }}$. Отметим, что из последнего равенства вытекает, что $H_{\text {ext }} \neq \varnothing$, так как множество $H_{\text {со }} \neq \varnothing$ (см. теорему 1$)$. Отметим также, что из определения отображения ext $\Phi$ (см. (37)) следует вложение $(\operatorname{ext} \Phi)(x) \subset \Phi(x)$, поэтому $H_{\text {ext }}(U) \subset H(U) \subset H_{\text {со }}(U)$.

Пусть $\left\{u_{m}\right\} \subset L^{n}[a, b]-$ последовательность, плотная в $\operatorname{co} \Phi\left(H_{\text {со }}(U)\right)$. Для каждых $m, i=1,2, \ldots$ определим отображения $\rho_{m}: H_{\text {со }}(U) \rightarrow \mathbb{R}^{1}$ и $\Phi_{m i}: H_{\mathrm{co}}(U)$ $\rightarrow \Omega\left(L^{n}[a, b]\right)$ равенствами

$$
\left.\rho_{m}(x)=\rho_{L^{n}[a, b]}\left[u_{m} ; \overline{\operatorname{co}}(\Phi(x))\right], \quad \Phi_{m i}=\overline{\operatorname{co} \Phi(x) \cap B_{L^{n}[a, b]}\left[u_{m} ; \rho_{m}(x)+i^{-1}\right.}\right] .
$$

Так как произведение $V$ соФ обладает свойством А, то отображение со $\Phi$ непрерывно на $U$. Поэтому на $H_{\text {со }}(U)$ непрерывны функционалы $\rho_{m}, m=1,2, \ldots$ Отсюда согласно $[6$, гл. $0, \S 3]$ отображения $\Phi_{m i}, m, i=1,2, \ldots$, полунепрерывны снизу на $H_{\text {со }}(U)$. Следовательно, согласно теореме Майкла [4] у $\Phi_{m i}$ сушествуют непрерывные ветви $g_{m i}: H_{\text {со }}(U) \rightarrow L^{n}[a, b], m, i=1,2, \ldots$.

Покажем, что последовательность $\left\{g_{m i}\right\}$ плотна в $\operatorname{co} \Phi(x)$ при любом $x \in$ $H_{\text {со }}(U)$. Возьмем призвольные $\varepsilon>0, x \in H_{\text {со }}(U), u \in \overline{\text { со }}(\Phi(x))$ и выберем такие $m, i=1,2, \ldots$, что выполняются неравенства $\left\|u_{m}-u\right\|_{L^{n}[a, b]} \leqslant 1 / i \leqslant \varepsilon / 4$. Тогда $u \in \overline{B_{L^{n}[a, b]}\left[u_{m} ; \rho_{m}(x)+i^{-1}\right]}$ причем согласно выбору чисел $m, i$ выполняется оценка $\rho_{m}(x)+1 / i \leqslant \varepsilon / 2$ и, следовательно, $\left\|u-g_{m i}\right\|_{L^{n}[a, b]}<\varepsilon$. Таким образом, последовательность $\left\{g_{m i}\right\}$ плотна в со $\Phi(x)$ при любом $x \in H_{\text {со }}(U)$.

Далее последовательность $\left\{g_{m i}\right\}$ будем обозначать через $\left\{g_{m}\right\}$, а последовательности функционалов $\left\{\eta\left(\cdot, g_{m}(\cdot)\right)\right\},\left\{\Theta\left(\cdot, g_{m}(\cdot)\right)\right\}$, определенные, соответственно, равенствами (44), (49) при $g=g_{m}, m=1,2, \ldots$, через $\left\{\eta_{m}\right\},\left\{\Theta_{m}\right\}$.

Для каждого $m, i=1,2, \ldots$ определим множества $H_{m i}^{1}, H_{m}, H_{m i}$, содержащиеся в $H_{\text {со }}(U)$, следующим образом:

$$
\begin{gathered}
H_{m i}^{1}=\left\{x \in H_{\mathrm{co}}(U): \Theta_{m}(x) \geqslant \frac{1}{i}\right\}, \\
H_{m}=\left\{x \in H_{\mathrm{co}}(U): \eta_{m}(x) \leqslant \frac{1}{2} \Theta_{m}(x)\right\}, \\
H_{m i}=H_{m i}^{1} \cap H_{m} .
\end{gathered}
$$


Покажем, что множества $H_{m i}$ замкнуты для любых $m, i=1,2, \ldots$ Прежде всего, согласно лемме 10 из [1] функционал $\Theta_{m}$ полунепрерывен сверху на $H_{\text {со }}(U)$. Поэтому множества $H_{m i}^{1}$ замкнуты при любых $m, i=1,2, \ldots$.

Теперь покажем, что множество $H_{m}$ замкнуто для любого $m=1,2, \ldots$ Пусть последовательность $\left\{x_{i}\right\} \subset H_{m}$ такова, что $x_{i} \rightarrow x \in H_{\text {со }}(U)$ в $C^{n}[a, b]$ при $i \rightarrow \infty$. Тогда согласно лемме 3 и лемме 10 из [1] получаем неравенства

$$
\eta_{m}(x) \leqslant \lim _{i \rightarrow \infty} \inf \eta_{m}\left(x_{i}\right) \leqslant \lim _{i \rightarrow \infty} \frac{1}{2} \sup \Theta_{m}\left(x_{i}\right) \leqslant \frac{1}{2} \Theta_{m}(x) .
$$

Следовательно, $x \in H_{m}$ (см. (50)) и множество $H_{m}$ замкнуто. Таким образом, замкнуто и множество $H_{m i}$ при любых $m, i=1,2, \ldots$ (см. (51)).

Далее, покажем, что множество $H_{\text {со }} \backslash H_{m j}$ плотно в $H_{\text {со }}(U)$ для любых $m, j=$ $1,2, \ldots$ Для этого достаточно показать, что $H_{m j} \subset \overline{H_{\text {со }}(U) \backslash H_{m j}}$. Пусть $\varepsilon>0$ и $x \in H_{m j}$ зафиксированы. Покажем, что найдется такой $y \in H_{\text {со }} \backslash H_{m j}$, для которого выполняется неравенство $\|x-y\|_{C^{n}[a, b]}<\varepsilon$.

Прежде всего, согласно следствию 4 и определению квазирешения включения (39) для выбранного $x \in H_{m j}$ найдется $v \in \Psi(x)$ и последовательность $z_{i} \in(\operatorname{ext} \Phi)(x), i=1,2, \ldots$, такие, что $y_{i}=v+V z_{i} \rightarrow x$ в $C^{n}[a, b]$ при $i \rightarrow \infty$. Отметим, что в силу условия теоремы $y_{i} \in U, i=1,2, \ldots$ Покажем, что в определении последовательности $y_{i}, i=1,2, \ldots$, функция $v=\sigma(x)$ (определение отображения $\sigma$ см. вьше). Действительно, не уменьшая обшности, можно считать, что $z_{i} \rightarrow z_{0} \in \operatorname{co} \Phi(x)$ слабо в $L^{n}[a, b]$ при $i \rightarrow \infty$. Поэтому $x=v+V z_{0}$ и, следовательно, в силу определения разложимости множества $H_{\mathrm{co}}(U)$ и определения отображения $\sigma$ получаем равенство $\sigma(x)=v$. Таким образом, для каждого $i=1,2, \ldots y_{i}=\sigma(x)+V z_{i}$.

Далее, пусть для любого $i=1,2, \ldots$ функция $\widetilde{y_{i}} \in \Psi\left(y_{i}\right)$ удовлетворяет равенству

$$
\left\|\sigma(x)-\widetilde{y}_{i}\right\|_{C^{n}[a, b]}=\rho_{C^{n}[a, b]}\left[\sigma(x) ; \Psi\left(y_{i}\right)\right] .
$$

Так как отображение $\Psi$ непрерывно на $U$, то из определения последовательности $\widetilde{y_{i}}, i=1,2, \ldots$, следует, что $\widetilde{y_{i}} \rightarrow \sigma(x)$ в $C^{n}[a, b]$ при $i \rightarrow \infty$.

Представим для любого $i=1,2, \ldots$ функцию $y_{i}$ в виде

$$
y_{i}=\widetilde{y_{i}}+V z_{i}+e_{i}
$$

где $e_{i}=\sigma(x)-\widetilde{y_{i}}$. Отметим, что $e_{i} \rightarrow 0$ в $C^{n}[a, b]$ при $i \rightarrow \infty$. Так как отображение $\Psi$ и произведение $V \operatorname{co} \Phi$ обладают свойством А на множестве $U$, то из неравенства (9) для любого измеримого множества $\mathscr{U} \subset[a, b]$ выполняется оценка

$$
\rho_{L^{n}(\mathscr{U})}\left[z_{i} ; \operatorname{co} \Phi\left(y_{i}\right)\right] \leqslant h_{L^{n}(\mathscr{U})}\left[\operatorname{co} \Phi(x) ; \operatorname{co} \Phi\left(y_{i}\right)\right] \leqslant \int_{\mathscr{U}}\left(\Gamma_{1}\left(Z\left(x-y_{i}\right)\right)\right)(s) d s,
$$

где $Z$ определено равенством (12). Из неравенств (52), а также из следствия 1 вытекает, что для каждой пары функций $\left(y_{i}, z_{i}\right), i=1,2, \ldots$, найдется решение $x_{i}=\sigma\left(x_{i}\right)+V z_{i}, \sigma\left(x_{i}\right) \in \Psi\left(x_{i}\right), \overline{z_{i}} \in \operatorname{co} \Phi\left(x_{i}\right)$, которое обладает следуюшим свойством: при любом $t \in[a, b]$ выполняется неравенство

$$
\left|x_{i}(t)-y_{i}(t)\right| \leqslant \xi_{i}(t)
$$


и при почти всех $t \in[a, b]$ справедлива оценка

$$
\left|z_{i}(t)-\overline{z_{i}}(t)\right| \leqslant \Gamma_{1}\left(Z\left(x-y_{i}\right)\right)(t)+\left(\Gamma_{1} \xi_{i}\right)(t)
$$

где $\xi_{i}$ - решение уравнения (13) с функцией $\nu=\nu_{i}$, при любом $t \in[a, b]$ определенной равенством

$$
\nu_{i}(t)=\int_{a}^{b}|V(t, s)|\left(\Gamma_{1}\left(Z\left(x-y_{i}\right)\right)\right)(s) d s+\left|e_{i}(t)\right| .
$$

Так как отображение $\Gamma_{1}$ непрерывно и удовлетворяет равенству $\Gamma_{1}(0)=0$, то $\nu_{i} \rightarrow 0$ в $C^{1}[a, b]$ при $i \rightarrow \infty$. Отсюда и из определения свойства А вытекает, что $\xi_{i} \rightarrow 0$ в $C^{1}[a, b]$ при $i \rightarrow \infty$. Следовательно, согласно неравенству (54) получаем $z_{i}-\overline{z_{i}} \rightarrow 0$ в $L^{n}[a, b]$ при $i \rightarrow \infty$. Так как при каждом $i=1,2, \ldots z_{i} \in(\operatorname{ext} \Phi)(x)$, то для каждого $i=1,2, \ldots$ справедливо неравенство

$$
\rho_{L^{n}[a, b]}\left[\overline{z_{i}} ;(\operatorname{ext} \Phi)(x)\right] \leqslant\left\|z_{i}-\overline{z_{i}}\right\|_{L^{n}[a, b]} \cdot
$$

Это означает, что

$$
\lim _{i \rightarrow \infty} \rho_{L^{n}[a, b]}\left[\overline{z_{i}} ;(\operatorname{ext} \Phi)(x)\right]=0 .
$$

Так как $x \in H_{m j}$, то $\Theta(x)>0$ (см. (49)-(51)). Отсюда и согласно равенству (55) найдутся такие числа $\delta>0$ и $i_{1}$, что при всех $i \geqslant i_{1}$ справедлива оценка

$$
\rho_{L^{n}[a, b]}\left[\overline{z_{i}} ;(\operatorname{ext} \Phi)(x)\right]<\frac{1}{2} \Theta_{m}(x)-\delta .
$$

Поэтому при всех $i \geqslant i_{1}$ получаем неравенство

$$
\begin{aligned}
\Theta_{m}(x) & \leqslant\left\|\overline{z_{i}}-g_{m}(x)\right\|_{L^{n}[a, b]}+\rho_{L^{n}[a, b]}\left[\overline{z_{i}} ;(\operatorname{ext} \Phi)(x)\right] \\
& <\left\|\overline{z_{i}}-g_{m}(x)\right\|_{L^{n}[a, b]}+\frac{1}{2} \Theta_{m}(x)-\delta
\end{aligned}
$$

Таким образом, при всех $i \geqslant i_{1}$ имеем оценку

$$
\left\|\overline{z_{i}}-g_{m}(x)\right\|_{L^{n}[a, b]}>\frac{1}{2} \Theta_{m}(x)+\delta
$$

Следовательно, при всех $i \geqslant i_{1}$ справедливо соотношение

$$
\overline{z_{i}} \notin \overline{B_{L^{n}[a, b]}\left[g_{m}(x) ; \frac{1}{2} \Theta_{m}(x)+\delta\right]} .
$$

В силу непрерывности $g_{m}$ и полунепрерывности сверху функционала $\Theta_{m}$ существует такое число $i_{2}$, что при всех $i \geqslant i_{2}$ выполняется вложение

$$
\overline{B_{L^{n}[a, b]}\left[g_{m}\left(x_{i}\right) ; \frac{1}{2} \Theta_{m}\left(x_{i}\right)\right]} \subset \overline{B_{L^{n}[a, b]}\left[g_{m}(x) ; \frac{1}{2} \Theta_{m}(x)+\delta\right]}
$$

Поэтому из $(56),(57)$ при всех $i \geqslant \max \left\{i_{1} ; i_{2}\right\}$ получаем

$$
\overline{z_{i}} \notin \overline{B_{L^{n}[a, b]}\left[g_{m}\left(x_{i}\right) ; \frac{1}{2} \Theta_{m}\left(x_{i}\right)\right]},
$$


т.е. при всех $i \geqslant \max \left\{i_{1} ; i_{2}\right\}$ вьполняется оценка $\eta\left(x_{i}\right)>\frac{1}{2} \Theta_{m}\left(x_{i}\right)$. Это означает, что $x_{i} \notin H_{m}$ (см. (50)). И поэтому $x_{i} \notin H_{m j}$ при всех $i \geqslant \max \left\{i_{1} ; i_{2}\right\}$ (см. (51)). Так как $\xi_{i} \rightarrow 0$ в $C^{1}[a, b]$, а $y_{i} \rightarrow x$ в $C^{n}[a, b]$ при $i \rightarrow \infty$, то из неравенства (53) вытекает, что $x_{i} \rightarrow x$ в $C^{n}[a, b]$ при $i \rightarrow \infty$. Поэтому за $y \notin H_{m j}$ можно взять такой элемент $x_{\bar{i}}, \bar{i} \geqslant \max \left\{i_{1} ; i_{2}\right\}$, для которого выполняется неравенство $\| x-$ $x_{\bar{i}} \|_{C^{n}[a, b]}<\varepsilon$. Таким образом, $H_{m j} \subset \overline{H_{\text {со }}(U) \backslash H_{m j}}$ и множество $H_{\text {со }}(U) \backslash H_{m j}$ плотно в $H_{\text {со }}(U)$.

Далее, покажем, что справедливо вложение

$$
\bigcap_{m, j=1}^{\infty}\left[H_{\mathrm{co}}(U) \backslash H_{m j}\right] \subset H_{\mathrm{ext}}(U) .
$$

Действительно, пусть $x \in H_{\text {со }}(U) \backslash H_{\text {ext }}(U)$ и $x=\sigma(x)+V z, \sigma(x) \in \Psi(x)$, $z \in \operatorname{co} \Phi(x)$. Тогда $z \notin(\operatorname{ext} \Psi)(x)$ и, следовательно, $\rho_{L^{n}[a, b]}[z ;(\operatorname{ext} \Phi)(x)]=\gamma>0$. Покажем, что $x \in H_{m i}$ при $1 / i \leqslant \frac{2}{3} \gamma$ и при $m$, для которого выполняется неравенство

$$
\eta_{m}(x)<\frac{\gamma}{3}
$$

где $\eta_{m}(x)=\left\|z-g_{m}(x)\right\|_{L^{n}[a, b]}, z=V^{-1}(x-\sigma(x))$. Такое $m$ сушествует, поскольку по построению последовательность $\left\{g_{m}(x)\right\}$ плотна в $\operatorname{co} \Phi(x)$. Согласно определению функционала $\Theta_{m}$ (см. (49)) и неравенству (59) получаем оценку

$$
\gamma \leqslant\left\|g_{m}(x)-z\right\|_{L^{n}[a, b]}+\Theta_{m}(x)<\frac{\gamma}{3}+\Theta_{m}(x) .
$$

Отсюда получаем неравенство

$$
\Theta_{m}(x)>\frac{2}{3} \gamma
$$

Из оценок (59), (60) вытекает соотношение $\eta_{m}(x)<\frac{1}{2} \Theta_{m}(x)$. Это означает, что $x \in H_{m}$ (см. (49)). Кроме того, так как $1 / i \leqslant \frac{2}{3} \gamma$, то из (60) следует неравенство $\Theta_{m}(x)>1 / i$, т.е. $x \in H_{m j}^{1}$ (см. (50)) и поэтому $x \in H_{m i}$ (см. (51)). Таким образом,

$$
x \notin \bigcap_{m, i=1}^{\infty}\left[H_{\mathrm{co}}(U) \backslash H_{m i}\right]
$$

и поэтому вложение (58) справедливо.

Так как множество $H_{\text {со }}(U)$ замкнуто, то, применяя теорему Бэра о категориях (см. $\left[7\right.$, гл. I, 2]) к последовательности открытых плотных в $H_{\text {со }}(U)$ множеств $H_{\text {со }}(U) \backslash H_{m i}$, получаем соотношение

$$
\overline{\bigcap_{m, i=1}^{\infty}\left[H_{\mathrm{co}}(U) \backslash H_{m i}\right]}=H_{\mathrm{co}}(U) .
$$

Из соотношений (58) и (61) вытекает, что $H_{\text {со }}(U)=\overline{H_{\text {ext }}(U)}$. Теорема доказана.

Из теоремы 6 вытекает

СлЕДСтвИЕ 8. Пусть отображсение $\Psi$ и произведение $V \operatorname{co\Phi ~обладают~}$ свойствами $\mathrm{A} u\left(V \Gamma_{2} ; P_{2}\right)$. Тогда $H_{\mathrm{ext}} \neq \varnothing u \overline{H_{\mathrm{ext}}}=H_{\mathrm{co}}$, где $\overline{H_{\mathrm{ext}}}-$ замыкание множества $H_{\mathrm{ext}}$ в пространстве $C^{n}[a, b]$.

ЗАмЕчАниЕ 3 . Отметим, что утверждения в $\S 1$ обобшают и уточняют результаты работ [1], [5], [8]-[14] по интегральньм и дифференциальным включениям. 


\section{§2. Краевые задачи дифференциальных включений с многозначным вектор-функционалом}

Здесь применяется рассмотренная в 11 теория для исследования свойств решений краевых задач для дифференциальных включений с невыпуклой правой частью и выпуклозначньм вектор-функционалом.

2.1. Функционально-дифференциальные включения. Рассмотрим краевую задачу

$$
\mathscr{L} x \in \Phi(x), \quad l x \in \varphi(x),
$$

где $\Phi: C^{n}[a, b] \rightarrow \Pi\left[L^{n}[a, b]\right], \varphi: C^{n}[a, b] \rightarrow \Omega\left(\mathbb{R}^{n}\right)$ - многозначные отображения; $l: D^{n}[a, b] \rightarrow \mathbb{R}^{n}, \mathscr{L}: D^{n}[a, b] \rightarrow L^{n}[a, b]$ - линейные непрерывные операторы. Пусть оператор $\Lambda: L^{n}[a, b] \rightarrow D^{n}[a, b]$ определен равенством

$$
(\Lambda z)(t)=\int_{a}^{t} z(s) d s, \quad t \in[a, b] .
$$

Запишем отображение $\mathscr{L}$ в виде (см. [16, гл. I, $\S 1.2])$

$$
\mathscr{L} x=Q \dot{x}+A(\cdot) x(a),
$$

где оператор $Q: L^{n}[a, b] \rightarrow L^{n}[a, b]$ (главная часть оператора $\mathscr{L}$ в представлении (63)) определяется равенством $Q=\mathscr{L} \Lambda$, каждый столбец $(n \times n)$-матрицы $A(t)$ представляет собой результат применения оператора $\mathscr{L}$ к соответствующему столбцу единичной матрицы: $A(t)=(\mathscr{L} E)(t)$. Будем предполагать, что оператор $Q$ имеет обратный и обратный оператор $Q^{-1}: L^{n}[a, b] \rightarrow L^{n}[a, b]$ непрерывен. Отметим, что этот класс линейных отображений содержит линейные дифференциальные операторы вида $(\mathscr{L} x)(t)=\dot{x}(t)+P(t) x(t)$, где $P(t)-(n \times n)$-матрицы с суммируемыми на $[a, b]$ элементами или дифференциальные отображения с операторами внутренней суперпозиции, или интегро-дифференциальные операторы и др. (см. [16, гл. I, §1.2]).

Под решением задачи (62) будем понимать такую функцию $x \in D^{n}[a, b]$, которая удовлетворяет и первому, и второму включениям в (62).

Далее, будем предполагать, что линейная однородная задача

$$
\mathscr{L} x=0, \quad l x=0
$$

имеет только нулевое решение. В этом случае согласно [16, гл. III, §3.1] сушествует непрерывный оператор Грина $G: L^{n}[a, b] \rightarrow D^{n}[a, b]$, определенный равенством

$$
(G z)(t)=\int_{a}^{b} G(t, s) z(s) d s, \quad t \in[a, b],
$$

который для произвольного $z \in L^{n}[a, b]$ решение $x \in D^{n}[a, b]$ краевой задачи

$$
\mathscr{L} x=z, \quad l x=0
$$

представляет в виде $x=G z$ и, наоборот, каждое значение $G z$-решение задачи (66). 
ЛЕммА 4. Краевая задача (62) эквивалентна интегральному включению

$$
x \in X(\cdot) \varphi(x)+G \Phi(x)
$$

где $X(\cdot)$ - фундаментальная матрица решений первого уравнения (64), удовлетворяющая условию $l(X)=E(E-$ единичная матрица, матрица $l(X)$ представляет собой результат применения вектор-функиионала $l$ к соответствующему столбиу матрицы X). Множсество решений включения (67) разложсимо.

ДокАЗАТЕльство. Действительно, пусть $x$ - решение включения (67). Тогда найдутся $c \in \varphi(x)$ и $z \in \Phi(x)$, для которых справедливо равенство $x=X(\cdot) c+G z$. Поэтому из выше приведенного свойства оператора Грина получаем, что $x-X(\cdot) c$ удовлетворяет равенствам $\mathscr{L}(x-X(\cdot) c)=z, l(x-X(\cdot) c)=0$. Так как $X(\cdot)$ - фундаментальная матрица решений первого уравнения (64), удовлетворяющая условию $l(X)=E$, то $\mathscr{L} x=z$ и $l x=c$, т.е. $x$ - решение задачи (62). Обратное утверждение доказывается аналогично.

Докажем теперь, что множество решений включения (67) разложимо (см. п. 1.2). Предположим противное, т.е. для решения $x$ включения (67) найдутся такие $c_{1}, c_{2} \in \varphi(x)$ и $z_{1}, z_{2} \in \Phi(x)$, для которых справедливы равенства

$$
x=X(\cdot) c_{1}+G z_{1}=X(\cdot) c_{2}+G z_{2}
$$

Отсюда получаем, что $X\left(c_{1}-c_{2}\right)=G\left(z_{1}-z_{2}\right)$ - решение задачи (66) для $z=$ $z_{2}-z_{1}$. Поэтому $l\left(X(\cdot)\left(c_{1}-c_{2}\right)\right)=0$ и отсюда вытекает, что $c_{1}=c_{2}$. Следовательно, $G\left(z_{2}-z_{1}\right)=0$ и поэтому $z_{1}=z_{2}$. Таким образом, множество решений включения (67) разложимо. Лемма доказана.

Далее, в силу леммы 4 , а также известных свойств оператора $G: L^{n}[a, b] \rightarrow$ $C^{n}[a, b]$ (см. [16, гл. III, $\left.\left.\S 3.1\right]\right)$ включение (67) удовлетворяет условиям, при которых рассматривалось включение (1). В связи с этим рассмотрим свойства решений задачи (62), вытекающие из изложенной в 11 теории.

Будем говорить, что многозначный функционал $\varphi$ и произведение $G \Phi$ обладают свойством $\left(G \widetilde{\Gamma}_{1} ; \widetilde{P}_{1}\right)^{\nu}$, если найдутся непрерывные изотонные отображения $\widetilde{\Gamma}_{1}: C_{+}^{1}[a, b] \rightarrow L^{1}[a, b]$ и $\widetilde{P}_{1}: C_{+}^{1}[a, b] \rightarrow[0 ; \infty)$, удовлетворяющие следующим условиям:

для любых $x, y \in C^{n}[a, b]$ и для любого измеримого множества $\mathscr{U} \subset[a, b]$ для отображения $\Phi$ выполняется неравенство (9), в котором $\Gamma_{1} \equiv \widetilde{\Gamma}_{1}$;

для любых $x, y \in C^{n}[a, b]$ для отображения $\widetilde{P}_{1}$ выполняется оценка

$$
h_{\mathbb{R}^{n}}[\varphi(x) ; \varphi(y)] \leqslant \widetilde{P}_{1}(Z(x-y))
$$

для непрерывного оператора $\widetilde{\mathfrak{A}}_{1}: C_{+}^{1}[a, b] \rightarrow C_{+}^{1}[a, b]$, определенного равенством

$$
\widetilde{\mathfrak{A}}_{1}(y)(t)=\int_{a}^{b}|G(t, s)|\left(\widetilde{\Gamma}_{1} y\right)(s) d s+\lambda \widetilde{P}_{1}(y)+\nu(t)
$$


сходятся последовательные приближения, где $\lambda=\max \{|X(t)|: t \in[a, b]\},|G(t, s)|$, $|X(t)|$ - согласованные с пространством $\mathbb{R}^{n}$ нормы $(n \times n)$-матриц $G(t, s)$ (в представлении (65)) и фундаментальной $X(t)$, соответственно, $\nu \in C_{+}^{1}[a, b]$, отображение $Z$ определено равенством (12).

Будем говорить, что многозначный функционал $\varphi$ и произведение $G \Phi$ обладают свойством $\left(G \widetilde{\Gamma}_{2} ; \widetilde{P}_{2}\right)$, если найдутся непрерывные изотонные отображения $\widetilde{\Gamma}_{2}: C_{+}^{1}[a, b] \rightarrow L^{1}[a, b]$ и $\widetilde{P}_{2}: C_{+}^{1}[a, b] \rightarrow[0 ; \infty)$, удовлетворяющие следуюшим условиям:

для любого $x \in C^{n}[a, b]$ и для любого измеримого множества $\mathscr{U} \subset[a, b]$ для отображения $\Phi$ выполняется неравенство $(26)$, в котором $\Gamma_{2} \equiv \widetilde{\Gamma}_{2}$;

для любого $x \in C^{n}[a, b]$ для отображения $\widetilde{P}_{2}$ выполняется оценка

$$
\|\varphi(x)\|_{\mathbb{R}^{n}} \leqslant \widetilde{P}_{2}(Z(x))
$$

для непрерывного оператора $\widetilde{\mathfrak{A}}_{2}: C_{+}^{1}[a, b] \rightarrow C_{+}^{1}[a, b]$, определенного равенством

$$
\widetilde{\mathfrak{A}}_{2}(y)(t)=\int_{a}^{b}|G(t, s)|\left(\widetilde{\Gamma}_{2} y\right)(s) d s+|X(t)| \widetilde{P}_{2}(y)
$$

сходятся последовательные приближения, где $|G(t, s)|,|X(t)|$ - согласованные с пространством $\mathbb{R}^{n}$ нормы $(n \times n)$-матриц $G(t, s)$ (в представлении $\left.(65)\right)$ и фундаментальной $X(t)$, соответственно, отображение $Z$ определено равенством (12).

Рассмотрим в пространстве $C^{1}[a, b]$ уравнение

$$
\xi_{\nu}(t)=\int_{a}^{b}|G(t, s)|\left(\widetilde{\Gamma}_{1} \xi_{\nu}\right)(s) d s+\lambda \widetilde{P}_{1}\left(\xi_{\nu}\right)+\nu(t)
$$

где число $\lambda$, функции $|G(t, s)|, \nu(t)$, отображения $\widetilde{\Gamma}_{1}, \widetilde{P}_{1}$ определены вьше. Согласно свойству $\left(G \widetilde{\Gamma}_{1} ; \widetilde{P}_{1}\right)^{\nu}$ решение уравнения (68) сушествует.

Пусть $\widetilde{q} \in C^{n}[a, b]$ и $w_{0} \in L^{n}[a, b]$. Представим функцию $\widetilde{q}$ равенством

$$
\widetilde{q}=X(\cdot) r_{0}+G w_{0}+e
$$

где $r_{0} \in \varphi(\widetilde{q}), e=\widetilde{q}-X(\cdot) r_{0}-G w_{0}$ (матрица $X(\cdot)$ удовлетворяет лемме 4 ). Пусть, далее, функция $\varkappa \in L^{1}[a, b]$ для каждого измеримого $\mathscr{U} \subset[a, b]$ удовлетворяет неравенству (15), а функция $\nu_{\varepsilon} \in C_{+}^{1}[a, b]$ для любого $t \in[a, b]$ определена соотношением

$$
\nu_{\varepsilon}(t)=\int_{a}^{b}|G(t, s)|(\varepsilon+\varkappa(s)) d s+\varepsilon+|e(t)|, \quad \varepsilon \geqslant 0
$$

где $|G(t, s)|$ - согласованная с пространством $\mathbb{R}^{n}$ норма $(n \times n)$-матрицы $G(t, s)$ в представлении (65), а $e$ - функция в правой части равенства (69).

Из леммы 4 и следствия 2 вытекает 
TеОРема 7. Пусть многозначный функционал $\varphi$ и произведение $G \Phi$ обладают свойствами $\left(G \widetilde{\Gamma}_{1} ; \widetilde{P}_{1}\right)^{\nu_{\varepsilon}},\left(G \widetilde{\Gamma}_{2} ; \widetilde{P}_{2}\right)$, әде функция $\nu_{\varepsilon}$ определена равенством (70). Тогда для любого $\varepsilon>0$ существует решение $x$ задачи (62), для которого при любом $t \in[a, b]$ выполняется неравенство $|x(t)-\widetilde{q}(t)| \leqslant \xi_{\varepsilon}(t)$ и при почти всех $t \in[a, b]$ выполняется оченка $\left|(\mathscr{L} x)(t)-w_{0}(t)\right| \leqslant \varepsilon+\varkappa(t)+\widetilde{\Gamma}_{1}\left(\xi_{\varepsilon}\right)(t)$, a также справедливо соотношение $\left\|X(\cdot)\left(r_{0}-l x\right)\right\|_{C^{n}[a, b]} \leqslant \lambda \widetilde{P}_{1}\left(\xi_{\varepsilon}\right)+\varepsilon$, где функции $\widetilde{q}, w_{0}$, вектор $r_{0}$ представимы равенством (69), $\xi_{\varepsilon}$ - решение уравнения (68) при $\nu=\nu_{\varepsilon}$, отображение $\widetilde{\Gamma}_{1}$ удовлетворяет неравенству (9) при

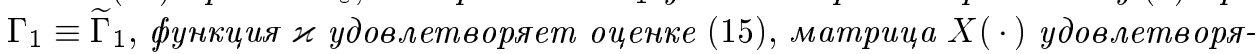
ет лемме 4, число $\lambda$ определено выше.

Если $\Phi: C^{n}[a, b] \rightarrow \Omega\left(\Pi\left[L^{n}[a, b]\right]\right)$, то утверждение справедливо и при $\varepsilon=0$.

Будем говорить, что многозначньй функционал $\varphi$ и произведение $G \Phi$ обладают свойством В, если вьполняются следующие условия:

найдется неотрицательная функция $\beta \in L^{1}[a, b]$ такая, что для любых $x, y \in$ $C^{n}[a, b]$ и любого измеримого множества $\mathscr{U} \in[a, b]$ выполняется неравенство

$$
h_{L^{n}(\mathscr{U})}[\Phi(x) ; \Phi(y)] \leqslant \int_{\mathscr{U}} \beta(s) d s\|x-y\|_{C^{n}[a, b]} ;
$$

найдется число $\alpha \geqslant 0$ такое, что для любых $x, y \in C^{n}[a, b]$ функционал $\varphi$ удовлетворяет неравенству

$$
h_{\mathbb{R}^{n}}[\varphi(x) ; \varphi(y)] \leqslant \alpha\|x-y\|_{C^{n}[a, b]} ;
$$

для функции $\beta \in L^{1}[a, b]$ и числа $\alpha \geqslant 0$ справедливо соотношение

$$
\max _{t \in[a, b]} \int_{a}^{b}|G(t, s)| \beta(s) d s+\alpha \lambda<1,
$$

где число $\lambda$, норма $(n \times n)$-матрищы $|G(t, s)|$ определены выше.

Рассмотрим в пространстве $C^{1}[a, b]$ уравнение

$$
\widetilde{\xi}_{\nu}(t)=\left(\int_{a}^{b}|G(t, s)| \beta(s) d s+\alpha \lambda\right)\left\|\widetilde{\xi}_{\nu}\right\|_{C^{1}[a, b]}+\nu(t),
$$

где функция $\nu \in C^{1}[a, b]$, число $\lambda$ определено выше, функция $\beta \geqslant 0$ и число $\alpha$ удовлетворяют неравенствам (71), (72), соответственно. Отметим, что согласно неравенству (73) уравнение (74) имеет решение для любой функции $\nu \in C_{+}^{1}[a, b]$.

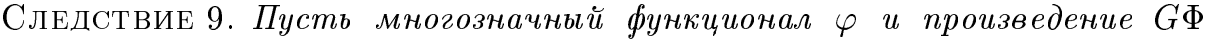
обладают свойством В. Тогда для любого $\varepsilon>0$ существует решение $x$ задачи (62), для которого при любом $t \in[a, b]$ выполняется неравенство $|x(t)-\widetilde{q}(t)| \leqslant \widetilde{\xi}_{\varepsilon}(t)$ и при почти всех $t \in[a, b]$ выполняется оценка $\left|(\mathscr{L} x)(t)-w_{0}(t)\right| \leqslant \varepsilon+\varkappa(t)+\beta(t)\left\|\widetilde{\xi}_{\varepsilon}\right\|_{C^{1}[a, b]}$, a также справедливо соотночение $\left\|X(\cdot)\left(r_{0}-l x\right)\right\|_{C^{n}[a, b]} \leqslant \lambda\left\|\widetilde{\xi}_{\varepsilon}\right\|_{C^{1}[a, b]}+\varepsilon$, где функции $\widetilde{q}, w_{0}$, вектор $r_{0}$ представимы равенством (69), $\widetilde{\xi}_{\varepsilon}-$ решение уравнения (74) при $\nu=\nu_{\varepsilon}$, 
которая определена равенством (70), функиии и, $\beta$ удовлетворяют неравенствам (15), (71), соответственно, матрица X(·) удовлетворяет лемме 4, число $\lambda$ определено выше.

Eсли $\Phi: C^{n}[a, b] \rightarrow \Omega\left(\Pi\left[L^{n}[a, b]\right]\right)$, то утвержсдение справедливо и при $\varepsilon=0$.

ДоказАТЕльство. Так как $\Phi(0) \in \Omega\left(\Pi\left[L^{n}[a, b]\right]\right)$, то найдется такая неотрицательная функция $\tau \in L^{1}[a, b]$, что для любого измеримого множества $\mathscr{U} \subset[a, b]$ вьполняется неравенство

$$
\|\Phi(0)\|_{L^{n}(\mathscr{U})} \leqslant \int_{\mathscr{U}} \tau(s) d s .
$$

Отсюда согласно неравенству (71) для любого $x \in C^{n}[a, b]$ и любого измеримого множества $\mathscr{U} \subset[a, b]$ имеем оценку

$$
\begin{aligned}
\|\Phi(x)\|_{L^{n}(\mathscr{U})}=h_{L^{n}(\mathscr{U})}[0 ; \Phi(x)] & \leqslant\|\Phi(0)\|_{L^{n}(\mathscr{U})}+h_{L^{n}(\mathscr{U})}[\Phi(0) ; \Phi(x)] \\
& \leqslant\left\|\widehat{\Gamma}_{2}(Z(x))\right\|_{L^{1}(\mathscr{U})}
\end{aligned}
$$

где $\widehat{\Gamma}_{2}(Z(x))(t)=\tau(t)+\beta(t)\|x\|_{C^{n}[a, b]}$. Далее, в силу неравенства (73) для отображения $\widehat{\mathfrak{A}}_{2}: C_{+}^{1}[a, b] \rightarrow C_{+}^{1}[a, b]$, заданного равенством

$$
\left(\widehat{\mathfrak{A}}_{2} z\right)(t)=\int_{a}^{b}|G(t, s)|\left(\widehat{\Gamma}_{2} z\right)(s) d s+|X(t)| \widehat{P}_{2}(z),
$$

где $\widehat{P}_{2}(z)=\alpha\|z\|_{C^{1}[a, b]}$, сходятся последовательные приближения. Поэтому функционал $\varphi$ и произведение $G \Phi$ обладают свойством $\left(G \widetilde{\Gamma}_{2} ; \widetilde{P}_{2}\right)$ при $\widetilde{\Gamma}_{2}=\widehat{\Gamma}_{2}$ и $\widetilde{P}_{2}=\widehat{P}_{2}$.

У оператора, определенного правой частью уравнения (74), в силу неравенства (73) сходятся последовательные приближения для любой функции $\nu \in C_{+}^{1}[a, b]$. Поэтому функционал $\varphi$ и произведение $G \Phi$ обладают свойством $\left(G \widetilde{\Gamma}_{1} ; \widetilde{P}_{1}\right)^{\nu_{\varepsilon}}$, в котором отображения $\widetilde{\Gamma}_{1}: C_{+}^{1}[a, b] \rightarrow L^{1}[a, b]$ и $\widetilde{P}_{1}: C_{+}^{1}[a, b] \rightarrow[0 ; \infty)$ определены соотношениями $\left(\widetilde{\Gamma}_{1} z\right)(t)=\beta(t)\|x\|_{C^{1}[a, b]},\left(\widetilde{P}_{1} z\right)=\alpha\|z\|_{C^{1}[a, b]}$. Таким образом, функционал $\varphi$ и произведение $G \Phi$ удовлетворяют условиям теоремы 7 . Следствие доказано.

ЗАмечАниЕ 4 . Если $\mathscr{L} x=\dot{x}, l x=x(a), \varphi(x)=x_{0}, x_{0} \in \mathbb{R}^{n}, \Phi$ - оператор Немыцкого, то оценки, установленњые в теореме 7 и следствии 9 , аналогичны оценкам, опубликованным в работах [17]-[20].

Рассмотрим вместе с задачей (62) и задачи

$$
\begin{array}{ll}
\mathscr{L} x \in \operatorname{co} \Phi(x), & l x \in \varphi(x), \\
\mathscr{L} x \in(\operatorname{ext} \Phi)(x), & l x \in \varphi(x),
\end{array}
$$

где многозначный оператор ext $\Phi: C^{n}[a, b] \rightarrow \Pi\left[L^{n}[a, b]\right]$ определен равенством (37). Пусть $H^{1}, H_{\text {co }}^{1}, H_{\mathrm{ext}}^{1}-$ множество всех решений задач $(62),(75),(76)$, соответственHо. 
Будем говорить, что многозначньй функционал $\varphi$ и произведение $G \Phi$ обладают свойством $\mathrm{C}$, если для любого $\varepsilon>0$ сушествует $\delta>0$, что для любой $\nu \in C_{+}^{1}[a, b]$, удовлетворяющей неравенству $\|\nu\|_{C^{1}[a, b]} \leqslant \delta$, выполняется свойство $\left(G \widetilde{\Gamma}_{1} ; \widetilde{P}_{1}\right)^{\nu}$, в котором для отображений $\widetilde{\Gamma}_{1}, \widetilde{P}_{1}$, справедливы равенства $\widetilde{\Gamma}_{1}(0)=0$, $\widetilde{P}_{1}(0)=0$, а решение уравнения (68) $\xi_{\nu}$ удовлетворяет неравенству $\left\|\xi_{\nu}\right\|_{C^{1}[a, b]}<\varepsilon$.

Так как согласно лемме 4 задачи $(75),(76)$ эквивалентны включениям

$$
x \in X(\cdot) \varphi(x)+G(\operatorname{co} \Phi(x)), \quad x \in X(\cdot) \varphi(x)+G((\operatorname{ext} \Phi)(x)),
$$

где $G$ - оператор Грина, $X(\cdot)$ - фундаментальная матрица решений первого уравнения (64), которые определены выше, то из леммы 4 и следствия 2 вытекает

ТЕОРема 8. Пусть многозначный функционал $\varphi$ и произведение $G \operatorname{co} \Phi$ об-

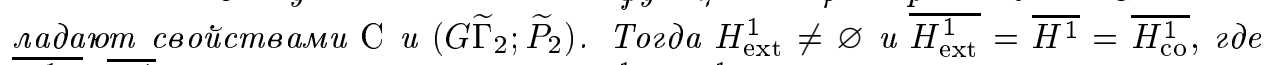
$\overline{H_{\mathrm{ext}}^{1}}, \overline{H^{1}}$ - замыкания множеств $H_{\mathrm{ext}}^{1}, H^{1}$ в пространстве $C^{n}[a, b]$, coответственно.

СлЕДСТВИЕ 10. Пусть многозначный функиионал $\varphi$ и произведение $G \operatorname{co} \Phi$ обладают свойством В. Тогда $H_{\mathrm{ext}}^{1} \neq \varnothing$ и $\overline{H_{\mathrm{ext}}^{1}}=\overline{H^{1}}=H_{\mathrm{co}}^{1}$, где $\overline{H_{\mathrm{ext}}^{1}}, \overline{H^{1}}-$ замыкания множеств $H_{\mathrm{ext}}^{1}, H^{1}$ в пространстве $C^{n}[a, b]$, соответственно.

Действительно, пусть $\widetilde{\xi}_{1}$ и $\widetilde{\xi}_{2}-$ решения уравнения (74) при $\nu=\nu_{1}$ и $\nu=\nu_{2}$, соответственно. Тогда из неравенства (73) вытекает оценка

$$
\left\|\widetilde{\xi}_{1}-\widetilde{\xi}_{2}\right\|_{C^{1}[a, b]} \leqslant \frac{\left\|\nu_{1}-\nu_{2}\right\|_{C^{1}[a, b]}}{1-\zeta}
$$

где $\zeta=\max _{t \in[a, b]} \int_{a}^{b}|G(t, s)| \beta(s) d s+\alpha \lambda,|G(t, s)|, \beta(s)$, числа $\alpha, \lambda$ определены выше. Поэтому решение уравнения (74) непрерывно зависит от параметра $\nu \in C_{+}^{1}[a, b]$. Следовательно, функционал $\varphi$ и произведение $G \operatorname{co} \Phi$ обладают свойством С.

Кроме того, из доказательства следствия 9 вытекает, что функционал $\varphi$ и произведение $G$ соФ обладают свойством $\left(G \widetilde{\Gamma}_{2} ; \widetilde{P}_{2}\right)$. Таким образом, следствие 10 удовлетворяет условиям теоремы 8.

ЗАмЕчАнИЕ 5. Отметим, что известные результаты о плотности множеств решений дифференциальных включений с невыпуклой и овыпукленной правыми частями и о “бэнг-бэнг" принципе (см. обзоры и монографии [21]-[27], а также работы [28]-[36]) касаются только задачи Коши для дифференциальных включений и предполагают в той или иной форме вольтерровость отображений $\mathscr{L}$ и $\Phi$. В теореме 8 и следствии 10 вольтерровость этих отображений не предполагается. Кроме того, эти утверждения распространяют результаты работ [1], [5], [9], [15] на случай, когда линейный вектор-функционал возмущен многозначным вектор-функционалом $\varphi$.

ЗАмечАниЕ 6. Отметим, что следствие 10 устанавливает, что для любого оператора Грина $G$, определенного равенством (65), и фундаментальной матрицы $X(\cdot)$ решений первого уравнения (64) найдутся достаточно малые функция 
$\beta \in L^{1}[a, b]$ и число $\alpha \geqslant 0$, удовлетворяющие неравенствам (71), (72), соответственно, для которых справедливо равенство $\overline{H_{\text {ext }}^{1}}=H_{\text {со }}$. То есть, другими словами, если задача (64) имеет только нулевое решение, то "бэнг-бэнг" принцип является устойчивым свойством относительно "малых" липшицевых возмушений, вызванных многозначным отображением $\Phi$ и многозначньм вектор-функционалом $\varphi$.

2.2. Дифференциальные включения с многозначным оператором Немыцкого. В этом случае можно получить мажорантные неравенства, параметры которых иногда представляют собой решения линейных интегральных уравнений.

Рассмотрим краевую задачу

$$
(\mathscr{L} x)(t) \in F(t, x(t)), \quad t \in[a, b], \quad l x \in \varphi(x),
$$

где линейные непрерывные операторы $\mathscr{L}: D^{n}[a, b] \rightarrow L^{n}[a, b], l: D^{n}[a, b] \rightarrow \mathbb{R}^{n}$ и многозначное отображение $\varphi: C^{n}[a, b] \rightarrow \Omega\left(\mathbb{R}^{n}\right)$ удовлетворяют условиям п. 2.1. Многозначная функция $F:[a, b] \times \mathbb{R}^{n} \rightarrow \operatorname{comp}\left[\mathbb{R}^{n}\right]$ удовлетворяет следующим условиям:

найдется неотрицательная функция $\beta \in L^{1}[a, b]$ такая, что для любых $x, y \in \mathbb{R}^{n}$ и при почти всех $t \in[a, b]$ выполняется неравенство

$$
h_{\mathbb{R}^{n}}[F(t, x) ; F(t, y)] \leqslant \beta(t)|x-y| ;
$$

для любого $x \in \mathbb{R}^{n}$ отображение $F(\cdot, x)$ измеримо;

функция $\|F(t, 0)\|_{\mathbb{R}^{n}}$ суммируема.

Напомним, что оператор Немыцкого $N: C^{n}[a, b] \rightarrow \Pi\left[L^{n}[a, b]\right]$, порожденньй функцией $F$, определяется равенством

$$
N x=\left\{y \in L^{n}[a, b]: y(t) \in F(t, x(t)) \text { при п.в. } t \in[a, b]\right\} .
$$

Будем говорить, что многозначный функционал $\varphi$ и произведение $G N$ обладают свойством $\widetilde{\mathrm{C}}^{\nu}$, если найдется число $\alpha \geqslant 0$ такое, что для любых $x, y \in$ $C^{n}[a, b]$ функционал $\varphi$ удовлетворяет неравенству (72), для непрерывного оператора $\widetilde{\mathfrak{A}}: C_{+}^{1}[a, b] \rightarrow C_{+}^{1}[a, b]$, определенного равенством

$$
(\widetilde{\mathfrak{A}} z)(t)=\int_{a}^{b}|G(t, s)| \beta(s) z(s) d s+\alpha \lambda\|z\|_{C^{1}[a, b]}+\nu(t),
$$

сходятся последовательные приближения. Здесь функция $\beta \in L^{1}[a, b]$ и число $\alpha$ удовлетворяют неравенствам (78) и (72), соответственно, $\lambda=\max \{|X(t)|$ : $t \in[a, b]\},|G(t, s)|,|X(t)|$ - согласованные с пространством $\mathbb{R}^{n}$ нормы $(n \times n)$-матриц $G(t, s)$ (в представлении $(65))$ и фундаментальной матрищы решений $X(\cdot)$ первого уравнения (64), соответственно, $\nu \in C_{+}^{1}[a, b]$.

Пусть оператор $\mathfrak{A}: C_{+}^{1}[a, b] \rightarrow C_{+}^{1}[a, b]$ определен равенством $(79)$ при $\nu=0$. Отметим, что если функционал $\varphi$ представляет собой постоянное множество из $\mathbb{R}^{n}$, то в этом случае $\alpha=0$ и тогда оператор $\mathfrak{A}$ представляет собой линейный интегральный оператор. Поэтому в этом случае, если спектральный радиус оператора $\mathfrak{A}$ меньше 1 , то для оператора $\widetilde{\mathfrak{A}}$, определенного равенством (79) (при $\alpha=0$ ), 
сходятся последовательные приближения для любой функции $\nu \in C_{+}^{1}[a, b]$. Если $\alpha>0$ в свойстве $\widetilde{\mathrm{C}}^{\nu}$, то в этом случае последовательные приближения оператора $\widetilde{\mathfrak{A}}$ сходятся, если, например, выполняется неравенство (73), в котором функция $\beta$ и произведение $\alpha \lambda$ определено в условии $\widetilde{\mathrm{C}}^{\nu}$.

Рассмотрим в пространстве $C^{1}[a, b]$ уравнение

$$
\psi_{\nu}(t)=\int_{a}^{b}|G(t, s)| \beta(s) \psi_{\nu}(s) d s+\alpha \lambda\left\|\psi_{\nu}\right\|_{C^{1}[a, b]}+\nu(t)
$$

Пусть функция $\widetilde{\nu} \in C_{+}^{1}[a, b]$ определена равенством

$$
\widetilde{\nu}(t)=\int_{a}^{b}|G(t, s)|\|F(t, 0)\|_{\mathbb{R}^{n}} d t
$$

где $|G(t, s)|$ определена выше.

ТЕОРема 9. Пусть функиионал $\varphi$ и произведение $G N$ обладают свойства-

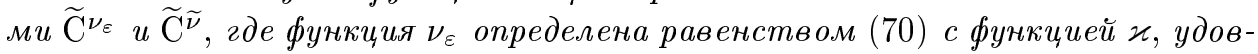
летворяющей неравенству (15) при $\Phi \equiv N$, а функиия $\widetilde{\nu}$ определена равенством (81). Тогда для любого $\varepsilon>0$ существует решение $x$ задачи (62), для которого при любом $t \in[a, b]$ выполняется неравенство $|x(t)-\widetilde{q}(t)| \leqslant \psi_{\varepsilon}(t)$, при почти всех $t \in[a, b]$ выполняется оценка $\left|(\mathscr{L} x)(t)-w_{0}(t)\right| \leqslant \varepsilon+\varkappa(t)+\beta(t) \psi_{\varepsilon}(t)$, a также справедливо соотношение $\left\|X(\cdot)\left(r_{0}-l x\right)\right\|_{C^{n}[a, b]} \leqslant \lambda\left\|\psi_{\varepsilon}\right\|_{C^{1}[a, b]}+\varepsilon$, где матрица $X(\cdot)$ удовлетворяет лемме 4, число $\lambda$ определено выше, функчии $\widetilde{q}, w_{0}$, вектор $r_{0}$ представимы равенством (69), $\psi_{\varepsilon}$ - решение уравнения (80) при $\nu=\nu_{\varepsilon}$, функиия $\beta$ удовлетворяет оценке (78), а для функиии $и$ справедливо соотношение (15), в котором $\Phi \equiv N$.

Eсли $N: C^{n}[a, b] \rightarrow \Omega\left(\Pi\left[L^{n}[a, b]\right]\right)$, то утверждение справедливо и при $\varepsilon=0$.

Действительно, в силу неравенства (78) для любого $x \in \mathbb{R}^{n}$ получаем оценку

$$
\|F(t, x)\|_{\mathbb{R}^{n}} \leqslant\|F(t, 0)\|_{\mathbb{R}^{n}}+\beta(t)|x|
$$

Далее, так как для оператора $\widetilde{\mathfrak{A}}$, определенного равенством (79) при $\nu=\widetilde{\nu}$ (функция $\widetilde{\nu}$ задана равенством $(81)$, сходятся последовательные приближения, то функционал $\varphi$ и произведение $G N$ обладают свойством $\left(G \widetilde{\Gamma}_{2} ; \widetilde{P}_{2}\right)$, в котором $\widetilde{\Gamma}_{2}$ и $\widetilde{P}_{2}$ определены равенствами $\left(\widetilde{\Gamma}_{2} z\right)(t)=\|F(t, 0)\|_{\mathbb{R}^{n}}+\beta(t) z(t), \widetilde{P}_{2} z=\alpha\|z\|_{C^{1}[a, b]}$. Таким образом, теорема 9 удовлетворяет условиям теоремы 7.

ЗАмечание 7 . Отметим, что если $\mathscr{L} x=\dot{x}$ и задача $(77)$ - задача Коши $\left(l x=x(a), \varphi(x)=x_{0}, x_{0} \in \mathbb{R}^{n}\right)$, то в этом случае задача (64) имеет вид $\dot{x}=0$, $x(a)=0$. Поэтому матрица $G(t, s)$, которая порождает оператор Грина задачи Коши, определяется равенствами $G(t, s)=E$ при $a \leqslant s \leqslant t \leqslant b$ и $G(t, s)=0$ при $a \leqslant t<s \leqslant b$, где $E$ - единичная матрица, а 0 - нулевая матрица. И, следовательно, в этом случае оператор $\widetilde{\mathfrak{A}}$, заданный равенством (79), в котором $\alpha=0$ и $\nu=0$, представляет собой оператор Вольтерра, у которого спектральньй радиус 
равен 0 для любой функции $\beta \in L^{1}[a, b]$, удовлетворяющей неравенству (78). Поэтому теорема 9 содержит в себе результат А. $\Phi$. Филиппова с точностью до $\varepsilon>0$ (см. [18]).

Рассмотрим краевые задачи

$$
\begin{aligned}
& (\mathscr{L} x)(t) \in \overline{\mathrm{co}}(F(t, x(t))), \quad t \in[a, b], \quad l x \in \varphi(x), \\
& (\mathscr{L} x)(t) \in \overline{\operatorname{ext}}(\overline{\operatorname{co}} F(t, x(t))), \quad t \in[a, b], \quad l x \in \varphi(x) .
\end{aligned}
$$

Пусть $H^{2}, H_{\text {co }}^{2}, H_{\text {ext }}^{2}$ - множество всех решений задачи $(77),(82),(83)$, соответственно. Пусть $M$ - многозначный оператор Немыцкого, порожденный функцией $\operatorname{co} F$.

Будем говорить, что функционал $\varphi$ и произведение $G N$ обладают свойством $\widetilde{\mathrm{C}}$, если для любого $\varepsilon>0$ сушествует $\delta>0$ такое, что для любой $\nu \in C_{+}^{1}[a, b]$, удовлетворяюшей неравенству $\|\nu\|_{C^{1}[a, b]} \leqslant \delta$, выполняется свойство $\widetilde{\mathrm{C}}^{\nu}$, причем решение уравнения (80) $\psi_{\nu}$ удовлетворяет неравенству $\left\|\psi_{\nu}\right\|_{C^{1}[a, b]} \leqslant \varepsilon$.

Отметим, что, если в свойстве $\widetilde{\mathrm{C}}^{\nu} \alpha=0$ (т.е. в этом случае $\varphi$-постоянное множество из $\mathbb{R}^{n}$ ), то функционал $\varphi$ и произведение $G N$ обладают свойством $\widetilde{\mathrm{C}}$, если спектральный радиус оператора $\widetilde{\mathfrak{A}}$, заданный равенством (79), в котором $\alpha=0$, $\nu=0$, меньше 1. Если в свойстве $\widetilde{\mathrm{C}}^{\nu} \alpha>0$, то многозначный функционал $\varphi$ и произведение $G N$ обладают свойством $\widetilde{\mathrm{C}}$, если, например, вьполняется неравенство (73).

Из теоремы 8 вытекает

ТЕОРема 10. Пусть многозначный функционал $\varphi$ и произведение $G M$ обладают свойствами $\widetilde{\mathrm{C}}$ и $\widetilde{\mathrm{C}} \widetilde{\nu}$, где функиия $\widetilde{\nu}$ определена равенством (81). Тогда $H_{\mathrm{ext}}^{2} \neq \varnothing, \overline{H_{\mathrm{ext}}^{2}}=\overline{H^{2}}=H_{\mathrm{co}}^{2}$, где $\overline{H_{\mathrm{ext}}^{2}}, \overline{H^{2}}-$ замыкания мнолеств $H_{\mathrm{ext}}^{2}, H^{2}$ в пространстве $C^{n}[a, b]$, соответственно.

\section{Список литературы}

1. Булгаков А.И. Интегральные включения с невьпукльми образами и их приложения к краевым задачам дифференциальных включений // Матем. сб. 1992. Т. 183. № 10. C. $63-86$.

2. Канторович Л. В., Акилов Г. П. Функциональный анализ. М.: Наука, 1977.

3. Иоффе А.Д., Тихомиров В. М. Теория экстремальных задач. М.: Наука, 1974.

4. Michael E. A. Selected selection theorems // Amer. Math. Monthly. 1956. V. 4. P. 233-236.

5. Булгаков А. И. Непрерывные ветви многозначных отображений и интегральные включения с невыпуклыми образами и их приложения. I // Дифференц. уравнения. 1992. Т. 28. № 3. C. 371-379.

6. Борисович Ю.Г., Гельман Б.Д., Мыикис А.Д., Обуховский В. В. Введение в теорию многозначных отображений. Воронеж: Изд-во ВГУ, 1985.

7. Обен ЖК.-П., Экланд И. Прикладной нелинейньй анализ. М.: Мир, 1988.

8. Булгаков А.И. Некоторые вопросы дифференциальных и интегральных включений с невьпуклой правой частью // Функционально-дифференциальные уравнения. Пермь: ППИ, 1991. С. 28-57.

9. Булгаков А. И. Непрерывные ветви многозначных отображений и интегральные включения с невьпукльпи образами и их приложения. II, III // Дифференц. уравнения. 1992. T. 28. № 4. C. 566-571; № 5. C. 739-746. 
10. Булгаков А.И., Васильева И. В. Сушествование решений включения Гаммерштейна с невьпукльми образами // Вестник ТГУ. 1996. Т. 1. № 2. С. 95-98.

11. Филиппов $A . \Phi$. Диффференциальные уравнения с разрывной правой частью. М.: Наука, 1985.

12. Ляпин Л. Н. Множественнозначные отображения в теории интегральных уравнений с разрьвным оператором // Дифференц. уравнения. 1973. Т. 9. № 8. С. 1511-1519.

13. Ляпин Л. Н. Непрерьвные решения интегральных включений // Дифференц. уравнения. 1974. Т. 10. №11. С. 2048-2055.

14. Ирисов A.E., Тонкова В. С., Тонков Е. Л. Периодические решения дифференщиального включения // Нелинейные колебания и теория управления. № 2. Ижевск: УдГУ, 1978. C. $3-15$.

15. Ирисов A.Е., Тонков Е.Л. О замыкании множества периодических решений дифференциального включения // Дифференциальные и интегральные уравнения. Горький: Изд-во ГГУ, 1983. С. 32-38.

16. Азбелев Н.В., Максимов В. П., Рахматуллина Л. Ф. Введение в теорию функционально-диффференциальных уравнений. М.: Наука, 1991.

17. Plis A. On trajectories of orientor fields // Bull. Acad. Pol. Sci. Ser. Sci. Math. Astron. Phys. 1965. V. 13. № 8. P. 571-573.

18. Филиппов $A . \Phi$. Классические решения дифференциальных уравнений с многозначной правой частью // Вестн. МГУ. Сер. 1. Матем., мех. 1967. № 3. С. 16-26.

19. Чугунов П.И. Свойства решений дифференциальных включений и управляемые системы // Прикладная математика и пакеты прикладных программ. Иркутск: Изд-во СЭИСО АН СССР, 1980. С. 155-179.

20. Толстоногов A.А., Чугунов П.И. О множестве решений дифференциального включения в банаховом пространстве // Сиб. матем. журн. 1983. Т. 24. №6. С. 144-159.

21. Благодатских В.И. Некоторые результаты по теории дифференциальных включений // Summer School on Ordinary Differential Equations. Crechoslovakia, Brno, 1974. Part II. P. 29-67.

22. Благодатских В.И., Филиппов А. Ф. Дифференциальные включения и оптимальное управление // Труды МИАН. 1985. Т. 169. С. 194-252.

23. Благодатских В. И. Теория диффференциальных включений. Часть І. М.: Изд-во МГУ, 1979.

24. Борисович Б. Г., Гельман Б.Д., Мьикис А. Д., Обуховский В.В. Многозначные отображения // Итоги науки и техники. Матем. анализ. Т. 19. М.: ВИНИТИ, 1982. C. $127-231$.

25. Половинкин E. С. Теория многозначных отображений. М.: МФТИ, 1982.

26. Субботин А.И., Ченцов А.Г. Оптимизация гарантии в задачах управления. М.: Наука, 1981.

27. Толстоногов A. А. Дифференциальные включения в банаховом пространстве. Новосибирск: Наука, 1986.

28. Булгаков А.И. Функционально-дифференциалњњые включения с невьпуклой правой частью // Дифференц. уравнения. 1990. Т. 26. №11. С. 1872-1878.

29. Суслов С. И. Нелинейньй бэнг-бэнг принцип I. Конечномерньй случай // Препринт. № 11. Новосибирск: Ин-т матем. СО АН СССР, 1989.

30. Суслов С.И. Нелинейный бэнг-бэнг принцип II. Бесконечномерньй случай // Препринт. № 12. Новосибирск: Ин-т матем. СО АН СССР, 1989.

31. Bressan A. On a bang-bang principle for nonlinear systems // Boll. Un. Mat. Ital. A(7). 1980. V. 1. P. 53-59.

32. Hermes $H$. On continuous and measurable selections and the existence of solutions of generalized differential equations // Proc. Amer. Math. Soc. 1971. V. 29. № 3. P. 535-542.

33. Hermes $H$. The generalized differential equation $\dot{x} \in R(t, x) / /$ Adv. Math. 1970. V. 4. № 2. P. 149-169.

34. Olech C. Lexicographical order, range of integrals and "Bang-bang" principle // Mathematical theory of control. New York: Acad. Press, 1967. P. 35-45. 
35. Papargeorgiou N. S. Functional-differential unclusions in Banach spaces with nonconvex right hand side // Funkcial. Ekvac. 1989. V. 32. P. 145-156.

36. Pianigiani G. On the fundamental theory of multivalued differential equations // J. Differential Equations. 1977. V. 25. № 1. P. 30-38.

37. Толстоногов А.А., Финогенко И. А. О решениях дифференциального включения с полунепрерывной снизу невыпуклой правой частью в банаховом пространстве // Матем. сб. 1984 . Т. 125 (167). № 2 (10). С. 199-230.

Тамбовский государственный университет

Поступила в редакцию 30.12 .1996 и 12.02 .1997 